\title{
Geometrically Uniform Frames
}

\author{
Yonina C. Eldar, Member, IEEE, and Helmut Bölcskei, Senior Member, IEEE
}

\begin{abstract}
We introduce a new class of finite-dimensional frames with strong symmetry properties, called geometrically uniform $(G U)$ frames, that are defined over a finite Abelian group of unitary matrices and are generated by a single generating vector. The notion of $\mathrm{GU}$ frames is then extended to compound $G U(C G U)$ frames which are generated by a finite Abelian group of unitary matrices using multiple generating vectors. The dual frame vectors and canonical tight frame vectors associated with GU frames are shown to be GU and, therefore, also generated by a single generating vector, which can be computed very efficiently using a Fourier transform (FT) defined over the generating group of the frame. Similarly, the dual frame vectors and canonical tight frame vectors associated with CGU frames are shown to be CGU. The impact of removing single or multiple elements from a GU frame is considered. A systematic method for constructing optimal GU frames from a given set of frame vectors that are not GU is also developed. Finally, the Euclidean distance properties of GU frames are discussed and conditions are derived on the Abelian group of unitary matrices to yield GU frames with strictly positive distance spectrum irrespective of the generating vector.
\end{abstract}

Index Terms-Compound geometrically uniform (CGU) frames, generalized Fourier transform (FT), geometrically uniform (GU) frames, least squares.

\section{INTRODUCTION}

$\mathbf{F}$ RAMES are generalizations of bases which lead to redundant signal expansions [1], [2]. A finite frame for a Hilbert space $\mathcal{H}$ is a set of vectors that are not necessarily linearly independent and span $\mathcal{H}$. Since the frame vectors can be linearly dependent, the conditions on frame vectors are usually not as stringent as the conditions on bases, allowing for increased flexibility in their design [3], [4].

Frames were first introduced by Duffin and Schaeffer [1] in the context of nonharmonic Fourier series, and play an important role in the theory of nonuniform sampling [1], [2], [5] and wavelet theory [3], [6]. Recently, frames have been used to analyze and design oversampled filter banks [7]-[9] and error-correction codes [10]. Frames have also been applied to the development of modern uniform and nonuniform sampling techniques [11], to various detection problems [12], [13], and to multiple description source coding [14].

Two important classes of highly structured frames are Gabor (Weyl-Heisenberg (WH)) frames [15], [16] and wavelet frames [3], [6], [17]. Both classes of frames are generated by a single

Manuscript received August 12, 2001; revised December 3, 2002.

Y. C. Eldar was with the Research Laboratory of Electronics, Massachusetts Institute of Technology, Cambridge, MA 02139 USA. She is now with the Technion-Israel Institute of Technology, Haifa 32000, Israel (e-mail: yonina@ee. technion.ac.il).

H. Bölcskei is with the Swiss Federal Institute of Technology (ETH) Zurich, Communication Technology Laboratory, CH-8092 Zurich (e-mail: boelcskei@nari.ee.ethz.ch).

Communicated by G. Battail, Associate Editor At Large.

Digital Object Identifier 10.1109/TIT.2003.809602 generating function. WH frames are obtained by translations and modulations of the generating function (referred to as the window function), and wavelet frames are obtained by shifts and dilations of the generating function (referred to as the mother wavelet). In Section III of this paper, we introduce a new class of frames which we refer to as geometrically uniform $(G U)$ frames, that like $\mathrm{WH}$ and wavelet frames are generated from a single generating vector. These frames are defined by a finite Abelian group $\mathcal{Q}$ of unitary matrices, referred to as the generating group of the frame. We note that WH frames and wavelet frames are, in general, not GU since the underlying group of matrices is, in general, not Abelian. GU frames are based on the notion of GU vector sets first introduced by Slepian [18] and later extended by Forney [19], which are known to have strong symmetry properties that may be desirable in various applications such as channel coding [19], [20], [21].

The notion of GU frames is then extended to frames that are generated by a finite Abelian group $\mathcal{Q}$ of unitary matrices using multiple generating vectors. Such frames are not necessarily GU, but consist of subsets of GU vector sets that are each generated by $\mathcal{Q}$. We refer to this class of frames as compound $G U$ (CGU) frames, and develop their properties in Section VI. CGU frames are a generalization of filter-bank frames introduced for $L_{2}(\mathbb{R})$ in [7]-[9]. An interesting class of frames results when the set of generating vectors is itself GU, generated by a finite Abelian group $\mathcal{G}$. (Note that this class of frames will in general not be GU.) As we show, these frames are a generalization of WH frames in which $\mathcal{Q}$ is the group of translations and $\mathcal{G}$ is the group of modulations.

Given a frame for $\mathcal{H}$, any signal in $\mathcal{H}$ can be represented as a linear combination of the frame vectors. However, if the frame vectors are linearly dependent, then the coefficients in this expansion are not unique. A popular choice of coefficients are the inner products of the signal with a set of analysis frame vectors called the dual frame vectors [17]. This choice of coefficients has the property that among all possible coefficients it has the minimal $l_{2}$-norm [17], [22].

In Section IV, we show that the dual frame vectors associated with a GU frame are also GU, and therefore generated by a single generating vector. Furthermore, we demonstrate that the generating vector can be computed very efficiently using a Fourier transform (FT) defined over the generating group $\mathcal{Q}$ of the frame. Similarly, in Section VI, we show that the dual-frame vectors associated with a CGU frame are also CGU. When the generating vectors of the CGU frame are GU and generated by a group $\mathcal{G}$ that commutes up to a phase factor with the group $\mathcal{Q}$, the dual frame is generated by a single generating vector, a result well known for WH frames.

An important topic in frame theory is the behavior of a frame when elements of the frame are removed. In Section VII, we 
show that the frame bounds of the frame resulting from removing a single vector of a $\mathrm{GU}$ frame are the same regardless of the particular vector removed. In this sense, GU frames exhibit an interesting robustness property which is of particular importance in applications such as multiple description source coding [23], [14]. We also consider the behavior of a GU frame when groups of frame elements are removed.

In the special case of a tight frame, the dual frame vectors are proportional to the original frame vectors so that the reconstruction formula is particularly simple. In many applications it is therefore desirable to construct a tight frame from an arbitrary set of frame vectors. A popular tight-frame construction is the so-called canonical tight frame [17], [7], [24]-[27], first proposed in the context of wavelets [28]. The canonical tight frame is relatively simple to construct, it is optimal in a least-squares sense [29], [27], [30], it can be determined directly from the given vectors, and plays an important role in wavelet theory [31]-[33]. Like the dual-frame vectors, we show that the canonical tight-frame vectors associated with a GU frame are GU, and the canonical tight-frame vectors associated with a CGU frame are CGU. When the generating vectors of the CGU frame are GU and generated by a group $\mathcal{G}$ that commutes up to a phase factor with $\mathcal{Q}$, the canonical tight-frame vectors can be obtained by a single generating vector, generalizing a result well-known in WH frame theory.

Since GU frames have nice symmetry properties, it may be desirable to construct such a frame from a given set of frame vectors. The problem of frame design has received relatively little attention in the literature. Systematic methods for constructing optimal tight frames have been considered [29], [27], [30]. Methods for generating frames starting from a given frame are described in [4].

In Section VIII, we systematically construct optimal GU frames from a given set of vectors, that are closest in a least-squares sense to the original frame vectors. The results in this section rely on ideas developed in [34] in the context of general least-squares inner product shaping. In our development, we consider three different constraints on the GU frame vectors. First, we treat the case in which the inner products of the frame vectors are known. The optimizing frame is referred to as the scaled-constrained least-squares GU frame (SC-LSGUF). Next, we consider the case where the inner products are known up to a scale factor. The optimizing frame in this case is referred to as the constrained least-squares GU frame (C-LSGUF). Finally, we consider the case in which both the inner products and the scaling are chosen to minimize the least-squares error between the original frame and the resulting tight frame. The optimizing frame is the least-squares GU frame (LSGUF).

In Section IX, we consider distance properties of GU frames, which may be of interest when using GU frames for code design (group codes) [18], [19]. In particular, we introduce a class of GU frames with strictly positive distance spectra for all choices of generating vectors. Such GU frames are shown to be generated by fixed-point-free groups [35].

Before proceeding to the detailed development, in Section II, we provide a brief introduction to frame expansions.



Fig. 1. Example of a frame. The vectors $\phi_{1}, \phi_{2}$, and $\phi_{3}$ span $\mathbb{R}^{2}$ and, therefore, form a frame for $\mathbb{R}^{2}$.

\section{FRAMES}

Frames, which are generalizations of bases, were introduced in the context of nonharmonic Fourier series by Duffin and Schaeffer [1] (see also [2]). Recently, the theory of frames has been expanded [3], [6], [17], [4], in part due to the utility of frames in analyzing wavelet decompositions.

Let $\left\{\phi_{i}, 1 \leq i \leq n\right\}$ denote a set of $n$ complex vectors in an $m$-dimensional Hilbert space $\mathcal{H}$. The vectors $\phi_{i}$ form a frame for $\mathcal{H}$ if there exist constants $A>0$ and $B<\infty$ such that ${ }^{1}$

$$
A\|x\|^{2} \leq \sum_{i=1}^{n}\left|\left\langle x, \phi_{i}\right\rangle\right|^{2} \leq B\|x\|^{2}
$$

for all $x \in \mathcal{H}$ [17]. In this paper, we restrict our attention to the case where $m$ and $n$ are finite. The lower bound in (1) ensures that the vectors $\phi_{i}$ span $\mathcal{H}$; thus, we must have $n \geq m$. Since $n<\infty$, the right-hand inequality of (1) is always satisfied with $B=\sum_{i=1}^{n}\left\|\phi_{i}\right\|^{2}$, so that any finite set of vectors that spans $\mathcal{H}$ is a frame for $\mathcal{H}$. In particular, any basis for $\mathcal{H}$ is a frame for $\mathcal{H}$. However, in contrast to basis vectors which are linearly independent, frame vectors with $n>m$ are linearly dependent. If the bounds $A=B$ in (1), then the frame is called a tight frame. If, in addition, $A=B=1$, then the frame is called a normalized tight frame. The redundancy of the frame is defined as $r=n / m$, i.e., $n$ vectors in an $m$-dimensional space.

A classical example of a frame is the frame depicted in Fig. 1. Since the vectors $\phi_{1}, \phi_{2}, \phi_{3}$ clearly span $\mathbb{R}^{2}$, they form a frame for $\mathbb{R}^{2}$. Note that the vectors are linearly dependent and therefore do not constitute a basis for $\mathbb{R}^{2}$. The frame of Fig. 1 has an interesting symmetry property: the frame vectors can be obtained by rotating any one of the vectors $\phi_{i}$ by multiples of $120^{\circ}$. As we will see in Section III, this frame is a cyclic frame which is a special case of a $\mathrm{GU}$ frame.

The frame operator corresponding to the frame vectors $\left\{\phi_{i}, 1 \leq i \leq n\right\}$ is defined as [17]

$$
S=\sum_{i=1}^{n} \phi_{i} \phi_{i}^{*}=\Phi \Phi^{*}
$$

\footnotetext{
${ }^{1}$ We use the notation $\langle x, y\rangle$ to denote the scalar product $x^{*} y$, where vectors $x$ and $y$ are represented as column vectors and $*$ denotes the Hermitian transpose.
} 
where $\Phi$ is the matrix of columns $\phi_{i}$, and (.)* denotes the Hermitian transpose. Using the frame operator, (1) can be rewritten as

$$
A\|x\|^{2} \leq\langle S x, x\rangle \leq B\|x\|^{2}
$$

for all $x \in \mathcal{H}$.

From (3), it follows that the tightest possible frame bounds $A$ and $B$ are given by $A=\min _{i} \lambda_{i}(S)$ and $B=\max _{i} \lambda_{i}(S)$, where $\left\{\lambda_{i}(S), 1 \leq i \leq m\right\}$ are the eigenvalues of the frame operator $S$. Throughout the paper, when referring to "the frame bounds" we implicitly assume the tightest possible frame bounds unless otherwise stated. Note that since the vectors $\phi_{i}$ span $\mathcal{H}, \Phi \Phi^{*}$ is invertible so that $A>0$.

If the vectors $\left\{\phi_{i}, 1 \leq i \leq n\right\}$ form a frame for $\mathcal{H}$, then any $x \in \mathcal{H}$ can be expressed as a linear combination of these vectors: $x=\sum_{i=1}^{n} a_{i} \phi_{i}$. If $n>m$, then the coefficients in this expansion are not unique. A possible choice is $a_{i}=\left\langle\bar{\phi}_{i}, x\right\rangle$ where $\bar{\phi}_{i}$ are the dual frame vectors [17] of the frame vectors $\phi_{i}$, and are given by

$$
\bar{\phi}_{i}=S^{-1} \phi_{i}
$$

The choice of coefficients $a_{i}=\left\langle\bar{\phi}_{i}, x\right\rangle$ has the property that among all possible coefficients it has the minimal $l_{2}$-norm [17], [22].

There are other choices of dual frame vectors $y_{i}$ such that for any $x \in \mathcal{H}, x=\sum_{i=1}^{n}\left\langle y_{i}, x\right\rangle \phi_{i}$. Specifically, with $Y$ denoting the matrix of columns $y_{i}$, any other choice corresponds to $Y$ of the form [36]

$$
Y=S^{-1} \Phi+T\left(I-\Phi^{*} S^{-1} \Phi\right)
$$

where $T$ is an arbitrary matrix. Indeed, $\Phi Y^{*}=I$ for any choice of $T$. However, the particular choice $y_{i}=\bar{\phi}_{i}$ has some desirable properties. Besides resulting in the minimal $l_{2}$-norm coefficients, in many cases the choice $y_{i}=\bar{\phi}_{i}$ yields frame vectors that share the same symmetries as the original frame vectors. Specifically, in Section IV we show that the dual frame vectors associated with a GU frame are also GU, and in Section VI we show that the dual frame vectors associated with a CGU frame are also CGU. Finally, in the case of a tight frame, the dual frame vectors lead to a particularly simple expansion. Specifically, in this case $S=A I_{m}$ so that $S^{-1}=(1 / A) I_{m}$, and the dual frame vectors are $\left\{\bar{\phi}_{i}=(1 / A) \phi_{i}, 1 \leq i \leq n\right\}$. Since a tight frame expansion of a signal is very simple, it is popular in many applications [17].

Suppose we are given a set of vectors $\left\{\phi_{i}, 1 \leq i \leq n\right\}$ that form a frame for $\mathcal{H}$, with frame bounds $A \neq B$. It may then be desirable to construct a tight frame from these vectors. A popular tight-frame construction is the canonical tight frame [17], [7], [24], [25], [27], [29], first proposed in the context of wavelets in [28]. The canonical tight-frame vectors $\left\{\mu_{i}, 1 \leq\right.$ $i \leq n\}$ associated with the vectors $\left\{\phi_{i}, 1 \leq i \leq n\right\}$ are given by

$$
\mu_{i}=S^{-1 / 2} \phi_{i}
$$

where $S^{-1 / 2}$ is the symmetric positive-definite square root of $S^{-1}$. Note, that $\left\{U S^{-1 / 2} \phi_{i}\right\}$ with $U$ an arbitrary unitary matrix yields a tight frame as well. The canonical tight frame, however, has the property that it is the closest normalized tight frame to the vectors $\left\{\phi_{i}\right\}$ in a least-squares sense [26], [29], [27].

From (4) and (6), we see that in order to compute the dualframe vectors and the canonical tight-frame vectors associated with a frame $\left\{\phi_{i}\right\}$, we need to compute the matrices $S^{-1}$ and $S^{-1 / 2}$ and then apply them to each of the frame vectors $\phi_{i}$. In the next section, we introduce a class of frames that have strong symmetry properties called $G U$ frames. As we show in Section IV, the dual frame vectors and the canonical tight frame vectors associated with a GU frame are generated by a single generating function, and can therefore be computed very efficiently.

\section{GU FRAMES}

\section{A. Definition}

A set of vectors $\mathcal{S}=\left\{\phi_{i}, 1 \leq i \leq n\right\}$ is GU [19], [18], [26] if every vector in the set has the form $\phi_{i}=U_{i} \phi$, where $\phi$ is an arbitrary generating vector and the matrices $\left\{U_{i}, 1 \leq i \leq n\right\}$ are unitary and form an Abelian group $2 \mathcal{Q}$. For concreteness, we assume that $U_{1}=I$ so that $\phi_{1}=\phi$. The group $\mathcal{Q}$ will be called the generating group of $\mathcal{S}$.

Alternatively, a vector set is GU if given any two vectors $\phi_{i}$ and $\phi_{j}$ in the set, there is an isometry (a norm-preserving linear transformation) $Z_{i j}$ that transforms $\phi_{i}$ into $\phi_{j}$ while leaving the set invariant [19]. Thus, for every $i, Z_{i j} \phi_{i}=\phi_{j}$. Intuitively, a vector set is GU if it "looks the same" geometrically from any of the points in the set.

The vector set of Fig. 1 is GU, since the set is symmetric with respect to a rotation by $120^{\circ}$. Further examples of GU vector sets are considered in [19].

A set of vectors $\left\{\phi_{i} \in \mathcal{H}, 1 \leq i \leq n\right\}$ forms a GU frame for $\mathcal{H}$ if the vectors $\left\{\phi_{i}\right\}$ are GU and span $\mathcal{H}$.

\section{B. Properties of GU Frames}

As we show in the following proposition, the frame bounds of a GU frame can be bounded by the norm of the generating vector.

Proposition 1: Let $\mathcal{S}=\left\{\phi_{i}=U_{i} \phi, U_{i} \in \mathcal{Q}\right\}$ be a GU frame with frame bounds $A$ and $B$, where $\phi$ is an arbitrary generating vector. Then, $A \leq \frac{n}{m}\|\phi\|^{2} \leq B$. If, in addition, the frame is tight, then $A=B=\frac{n}{m}\|\phi\|^{2}$.

Proof: We can express the frame operator corresponding to the frame vectors $\phi_{i}$ as

$$
S=\sum_{i=1}^{n} U_{i} \phi \phi^{*} U_{i}^{*} .
$$

Then

$$
\operatorname{Tr}(S)=\sum_{i=1}^{n} \operatorname{Tr}\left(U_{i} \phi \phi^{*} U_{i}^{*}\right)=n\|\phi\|^{2}
$$

\footnotetext{
${ }^{2}$ That is, $\mathcal{Q}$ contains the identity matrix $I$; if $\mathcal{Q}$ contains $U_{i}$, then it also contains its inverse $U_{i}^{-1}$; the product $U_{i} U_{j}$ of any two elements of $\mathcal{Q}$ is again in $\mathcal{Q}$; and $U_{i} U_{j}=U_{j} U_{i}$ for any two elements in $\mathcal{Q}$ [37].
} 
so that

$$
\sum_{i=1}^{m} \lambda_{i}(S)=\operatorname{Tr}(S)=n\|\phi\|^{2}
$$

Therefore,

$$
A=\min _{i} \lambda_{i}(S) \leq \frac{1}{m} \sum_{i=1}^{m} \lambda_{i}(S)=\frac{n}{m}\|\phi\|^{2}
$$

and

$$
B=\max _{i} \lambda_{i}(S) \geq \frac{1}{m} \sum_{i=1}^{m} \lambda_{i}(S)=\frac{n}{m}\|\phi\|^{2} .
$$

Since $U_{i}^{*}=U_{i}^{-1}$, the inner product of two vectors in $\mathcal{S}$ is

$$
\left\langle\phi_{i}, \phi_{j}\right\rangle=\phi^{*} U_{i}^{-1} U_{j} \phi=s\left(U_{i}^{-1} U_{j}\right)
$$

where $s$ is the function on $\mathcal{Q}$ defined by

$$
s\left(U_{i}\right)=\phi^{*} U_{i} \phi
$$

For fixed $i$, the set $U_{i}^{-1} \mathcal{Q}=\left\{U_{i}^{-1} U_{j}, U_{j} \in \mathcal{Q}\right\}$ is just a permutation of $\mathcal{Q}$ since $U_{i}^{-1} U_{j} \in \mathcal{Q}$ for all $i, j$ [37]. Therefore, the $n$ numbers $\left\{s\left(U_{i}^{-1} U_{j}\right), 1 \leq j \leq n\right\}$ are a permutation of the numbers $\left\{s\left(U_{i}\right), 1 \leq i \leq n\right\}$. The same is true for fixed $j$. Consequently, every row and column of the $n \times n$ Gram matrix $G=\left\{\left\langle\phi_{i}, \phi_{j}\right\rangle\right\}$ is a permutation of the numbers $\left\{a_{i}=s\left(U_{i}\right), 1 \leq i \leq n\right\}$.

A matrix $G$ whose rows (columns) are a permutation of the first row (column) will be called a permuted matrix. ${ }^{3}$ Thus, we have shown that the Gram matrix of a GU vector set is a permuted matrix. Furthermore, if the Gram matrix $G=\left\{\left\langle\phi_{i}, \phi_{j}\right\rangle\right\}$ is a permuted matrix and in addition $G=G^{T}$, then the vectors $\left\{\phi_{i}\right\}$ are GU [34]. We, therefore, have the following proposition.

Proposition 2: The Gram matrix $G=\left\{\left\langle\phi_{i}, \phi_{j}\right\rangle\right\}$ corresponding to a $\mathrm{GU}$ vector set $\mathcal{S}=\left\{\phi_{i} \in \mathcal{H}, 1 \leq i \leq n\right\}$ is a permuted matrix. Conversely, if the Gram matrix $G=\left\{\left\langle\phi_{i}, \phi_{j}\right\rangle\right\}$ is a permuted matrix, and $\left\langle\phi_{i}, \phi_{j}\right\rangle=\left\langle\phi_{j}, \phi_{i}\right\rangle$ for all $i, j$, then the vectors $\left\{\phi_{i}\right\}$ are GU. If, in addition, the vectors $\left\{\phi_{i}\right\}$ span $\mathcal{H}$, then they form a GU frame for $\mathcal{H}$.

As we will see in the sequel, the FT matrix plays an important role in defining GU frames. To define the FT it will be convenient to replace the multiplicative group $\mathcal{Q}$ by an additive group $Q$ to which $\mathcal{Q}$ is isomorphic. ${ }^{4}$ Specifically, it is well known (see, e.g., [37]) that every finite Abelian group $\mathcal{Q}$ is isomorphic to a direct product $Q$ of a finite number of cyclic groups: $\mathcal{Q} \cong Q=\mathbb{Z}_{n_{1}} \times \cdots \times \mathbb{Z}_{n_{p}}$, where $\mathbb{Z}_{n_{t}}$ is the cyclic additive group of integers modulo $n_{t}$, and $n=\prod_{t} n_{t}$. Thus, every ele-

${ }^{3} \mathrm{An}$ example of a permuted matrix is

$$
\left[\begin{array}{llll}
a_{1} & a_{2} & a_{3} & a_{4} \\
a_{2} & a_{1} & a_{4} & a_{3} \\
a_{3} & a_{4} & a_{1} & a_{2} \\
a_{4} & a_{3} & a_{2} & a_{1}
\end{array}\right] .
$$

${ }^{4}$ Two groups $\mathcal{Q}$ and $\mathcal{Q}^{\prime}$ are isomorphic, denoted by $\mathcal{Q} \cong \mathcal{Q}^{\prime}$, if there is a bijection (one-to-one and onto map) $\varphi: \mathcal{Q} \rightarrow \mathcal{Q}^{\prime}$ which satisfies $\varphi(x y)=$ $\varphi(x) \varphi(y)$ for all $x, y \in \mathcal{Q}$ [37]. ment $U_{i} \in \mathcal{Q}$ can be associated with an element $q \in Q$ of the form $q=\left(q_{1}, q_{2}, \ldots, q_{p}\right)$, where $q_{t} \in \mathbb{Z}_{n_{t}}$; this correspondence is denoted by $U_{i} \leftrightarrow q$.

Each vector $\phi_{i}=U_{i} \phi$ is then denoted as $\phi(q)$, where $U_{i} \leftrightarrow$ $q$. The zero element $0=(0,0, \ldots, 0) \in Q$ corresponds to the identity matrix $I \in \mathcal{Q}$, and an additive inverse $-q \in Q$ corresponds to a multiplicative inverse $U_{i}^{-1}=U_{i}^{*} \in \mathcal{Q}$. The Gram matrix is then the $n \times n$ matrix

$$
G=\left\{\left\langle\phi\left(q^{\prime}\right), \phi(q)\right\rangle, q^{\prime}, q \in Q\right\}=\left\{s\left(q-q^{\prime}\right), q^{\prime}, q \in Q\right\}
$$

with row and column indexes $q^{\prime}, q \in Q$, where $s$ is now the function on $Q$ defined by

$$
s(q)=\langle\phi(0), \phi(q)\rangle .
$$

The FT of a complex-valued function $\varphi: Q \rightarrow \mathbb{C}$ defined on $Q=\mathbb{Z}_{n_{1}} \times \cdots \times \mathbb{Z}_{n_{p}}$ is the complex-valued function $\hat{\varphi}: Q \rightarrow \mathbb{C}$ defined by

$$
\hat{\varphi}(h)=\frac{1}{\sqrt{n}} \sum_{q \in Q}\langle h, q\rangle \varphi(q)
$$

where the Fourier kernel $\langle h, q\rangle$ is

$$
\langle h, q\rangle=\prod_{t=1}^{p} e^{-2 \pi i h_{t} q_{t} / n_{t}} .
$$

Here, $h_{t}$ and $q_{t}$ are the $k$ th components of $h$ and $q$, respectively, and the product $h_{t} q_{t}$ is taken as an ordinary integer modulo $n_{t}$.

The FT matrix over $Q$ is defined as the $n \times n$ matrix

$$
\mathcal{F}=\left\{\frac{1}{\sqrt{n}}\langle h, q\rangle, h, q \in Q\right\} .
$$

The FT of a column vector $\varphi=\{\varphi(q), q \in Q\}$ is then the column vector $\hat{\varphi}=\{\hat{\varphi}(h), h \in Q\}$ given by $\hat{\varphi}=\mathcal{F} \varphi$. Since $\mathcal{F}$ is unitary, we obtain the inverse FT formula

$$
\varphi=\mathcal{F}^{*} \hat{\varphi}=\left\{\frac{1}{\sqrt{n}} \sum_{h \in Q}\langle h, q\rangle^{*} \hat{\varphi}(h), q \in Q\right\} .
$$

As we show in the following theorem, the FT matrix can be used to define $\mathrm{GU}$ frames.

Theorem 1: A set of vectors $\left\{\phi_{i}, 1 \leq i \leq n\right\}$ in an $m$-dimensional Hilbert space $\mathcal{H}$ is GU if and only if the Gram matrix $G=\left\{\left\langle\phi_{i}, \phi_{j}\right\rangle\right\}$ is diagonalized by an FT matrix $\mathcal{F}$ over a finite product of cyclic groups $Q$. The vectors $\left\{\phi_{i}\right\}$ form a GU frame for $\mathcal{H}$ if in addition $G$ has rank $m$.

Proof: The vectors $\left\{\phi_{i}\right\}$ form a frame for $\mathcal{H}$ if and only if they span $\mathcal{H}$, which implies that the rank of $G$, must be equal to $m$.

For a $\mathrm{GU}$ vector set with generating group $\mathcal{Q} \cong Q$, the $\mathrm{FT}$ over $Q$ diagonalizes the Gram matrix $G$ [26]. Thus, to complete the proof of the theorem, we need to prove that if $G$ is diagonalized by an FT matrix $\mathcal{F}$ over the group $Q$, then the vector set $\{\phi(q), q \in Q\}$ is GU.

Let $\Phi$ be the matrix of columns $\phi(q)$, so that $G=\Phi^{*} \Phi$. Since $\mathcal{F}$ diagonalizes $G, G$ has an eigendecomposition of the form 
$G=\mathcal{F} D \mathcal{F}^{*}$ for a diagonal matrix $D$ with diagonal elements $d_{i}$, where the first $m$ diagonal elements may be nonzero and the remaining diagonal elements are all zero. Then, $\Phi$ has a singular value decomposition (SVD) [38] of the form $\Phi=U \Sigma \mathcal{F}^{*}$, where $U$ is an arbitrary unitary matrix and $\Sigma$ is an $m \times n$ diagonal matrix with diagonal elements $\left\{\sigma_{i}=\sqrt{d_{i}}, 1 \leq i \leq m\right\}$.

Let $\{f(q), q \in Q\}$ denote the columns of $\mathcal{F}^{*}$. From the definition of $\mathcal{F}$, the components of $f(0)$ are all equal to $1 / \sqrt{n}$, and $f(q)=B(q) f(0)$ where $B(q)$ is a diagonal unitary matrix with diagonal elements $\{\langle h, q\rangle, h \in Q\}$, where $\langle h, q\rangle$ is given by (18). Then

$$
\begin{aligned}
\phi(q) & =U \Sigma f(q)=U \Sigma B(q) f(0) \\
& =U B(q) \Sigma f(0)=U B(q) U^{*} \phi
\end{aligned}
$$

where $\phi=U \Sigma f(0)$, and where we used the fact that diagonal matrices commute. If we now define $U(q)=U B(q) U^{*}$, then we have that $\phi(q)=U(q) \phi$, where the matrices $\{U(q), q \in Q\}$ are unitary.

We now show that the group $\mathcal{Q}=\{U(q), q \in Q\}$ is an Abelian group. First, we have that

$$
U(0)=U B(0) U^{*}=U U^{*}=I
$$

so that $I \in \mathcal{Q}$. Next

$$
U^{-1}(q)=U B^{-1}(q) U^{*}=U B(-q) U^{*}
$$

so that $U^{-1}(q) \in \mathcal{Q}$ since $-q \in Q$. Finally,

$$
U(q) U(h)=U B(q) B(h) U^{*}=U B(h) B(q) U^{*}=U(h) U(q)
$$

since diagonal matrices commute, and $U(q) U(h) \in \mathcal{Q}$ since $B(h) B(q)=B(h+q)=B\left(q^{\prime}\right)$ for some $q^{\prime} \in Q$.

We, therefore, conclude that $\phi(q)=U(q) \phi$ where the matrices $U(q)$ are unitary and form an Abelian group, so that the vectors $\phi(q)$ are GU. lary.

As a consequence of Theorem 1 we have the following corol-

Corollary 1: A set of vectors $\left\{\phi_{i} \in \mathcal{H}, 1 \leq i \leq n\right\}$ is GU if and only if the matrix $\Phi$ of columns $\phi_{i}$ has an SVD of the form $\Phi=U \Sigma \mathcal{F}^{*}$, where $U$ is an arbitrary unitary matrix, $\Sigma$ is an arbitrary diagonal matrix with diagonal elements $\left\{\sigma_{i}, 1 \leq\right.$ $i \leq m\}$, and $\mathcal{F}$ is an FT matrix over a direct product of cyclic groups. In addition, the vectors $\left\{\phi_{i}\right\}$ form a GU frame for $\mathcal{H}$ if they span $\mathcal{H}$ or, equivalently, if $\sigma_{i} \neq 0$ for $1 \leq i \leq m$.

\section{Dual and Canonical Tight Frames Associated WITH GU FRAMES}

In Section IV-A, we show that the dual-frame vectors and the canonical tight-frame vectors associated with a GU frame are also GU. This property can then be used to compute the dual and canonical tight frames very efficiently. Further properties of the canonical tight-frame vectors are discussed in Section IV-B.

\section{A. Constructing the Dual and Canonical Tight Frames}

Let $\mathcal{S}=\left\{\phi_{i}=U_{i} \phi, U_{i} \in \mathcal{Q}\right\}$ be a GU frame generated by a finite (not necessarily Abelian) group $\mathcal{Q}$ of unitary matrices, where $\phi$ is an arbitrary generating vector. Then the frame operator $S$ defined by (2) commutes with each of the unitary matrices $U_{i}$ in the generating group $\mathcal{Q}$. Indeed, expressing the frame operator as

$$
S=\sum_{i=1}^{n} U_{i} \phi \phi^{*} U_{i}^{*}
$$

we have that for all $j$

$$
\begin{aligned}
S U_{j} & =\sum_{i=1}^{n} U_{i} \phi_{i} \phi_{i}^{*} U_{i}^{*} U_{j} \\
& =U_{j} \sum_{i=1}^{n} U_{j}^{*} U_{i} \phi \phi^{*} U_{i}^{*} U_{j} \\
& =U_{j} \sum_{i=1}^{n} U_{i} \phi \phi^{*} U_{i}=U_{j} S
\end{aligned}
$$

since $\left\{U_{j}^{*} U_{i}, 1 \leq i \leq n\right\}$ is just a permutation of $\mathcal{Q}$.

If $S$ commutes with $U_{j}$, then $S^{-1}$ and $S^{-1 / 2}$ also commute with $U_{j}$ for all $j$. Thus,

$$
\bar{\phi}_{i}=S^{-1} \phi_{i}=S^{-1} U_{i} \phi=U_{i} S^{-1} \phi=U_{i} \bar{\phi}
$$

where $\bar{\phi}=S^{-1} \phi$, which shows that the dual frame vectors $\left\{\bar{\phi}_{i}=S^{-1} \phi_{i}\right\}$ are GU with generating group equal to $\mathcal{Q}$.

Similarly

$$
\mu_{i}=S^{-1 / 2} \phi_{i}=S^{-1 / 2} U_{i} \phi=U_{i} S^{-1 / 2} \phi=U_{i} \mu
$$

where $\mu=S^{-1 / 2} \phi$, which shows that the canonical tight frame vectors $\left\{\mu_{i}=S^{-1 / 2} \phi_{i}\right\}$ are also GU with generating group $\mathcal{Q}$.

Therefore, to compute the dual frame vectors or the canonical tight frame vectors all we need is to compute the generating vectors $\bar{\phi}$ and $\mu$, respectively. The remaining frame vectors are then obtained by applying the group $\mathcal{Q}$ to the corresponding generating vectors.

We now show that when the group $\mathcal{Q}$ is Abelian, the generating vectors can be computed very efficiently using the FT. From Corollary 1, we have that $\Phi$ has an SVD of the form

$$
\Phi=U \Sigma \mathcal{F}^{*}=\sum_{h \in Q} \sigma(h) u(h) \mathcal{F}^{*}(h) .
$$

Here, $\Sigma$ is a diagonal matrix with diagonal elements

$$
\left\{\sigma(h)=n^{1 / 4} \sqrt{\hat{s}(h)}, h \in Q\right\}
$$

where $\{\hat{s}(h), h \in Q\}$ is the FT of $\{s(q), q \in Q\}, U$ is the matrix of columns $u(h)$, where

$$
u(h)= \begin{cases}\Phi \mathcal{F}(h) / \sigma(h)=\hat{\phi}(h) / \sigma(h), & \text { if } \sigma(h) \neq 0 \\ 0, & \text { otherwise }\end{cases}
$$

with

$$
\hat{\phi}(h)=\frac{1}{\sqrt{n}} \sum_{q \in Q}\langle h, q\rangle \phi(q)
$$

denoting the $h$ th element of the FT of $\Phi$ regarded as a row vector of column vectors, $\Phi=\{\phi(q), q \in G\}$, and

$$
\mathcal{F}^{*}=\left\{\frac{1}{\sqrt{n}}\langle h, q\rangle^{*}, h, q \in Q\right\}
$$

has rows $\mathcal{F}^{*}(h)=\left\{\frac{1}{\sqrt{n}}\langle h, q\rangle^{*}, q \in Q\right\}$. 
It then follows that

$$
\bar{\phi}=S^{-1} \phi=\frac{1}{\sqrt{n}} \sum_{h \in \mathcal{I}} \frac{1}{\sigma(h)} u(h)
$$

where $h \in \mathcal{I}$ if $\sigma(h) \neq 0$. Similarly

$$
\mu=S^{-1 / 2} \phi=\frac{1}{\sqrt{n}} \sum_{h \in \mathcal{I}} u(h) .
$$

We summarize our results in the following theorem.

Theorem 2 (GU Frames): Let $\mathcal{S}=\left\{\phi_{i}=U_{i} \phi, U_{i} \in \mathcal{Q}\right\}$ be a GU frame generated by a finite Abelian group $\mathcal{Q}$ of unitary matrices, where $\phi$ is an arbitrary generating vector, and let $\Phi$ be the matrix of columns $\phi_{i}$. Let $Q$ be an additive Abelian group isomorphic to $\mathcal{Q}$, let $\{\phi(q), q \in Q\}$ be the elements of $\mathcal{S}$ under this isomorphism, and let $\mathcal{F}$ be the FT matrix over $G$. Then

1) the dual frame vectors $\left\{\bar{\phi}_{i}, 1 \leq i \leq n\right\}$ are GU with generating group $\mathcal{Q}$ and generating vector

$$
\bar{\phi}=(1 / \sqrt{n}) \sum_{h \in \mathcal{I}}(1 / \sigma(h)) u(h)
$$

where

a) $\left\{\sigma(h)=n^{1 / 4} \sqrt{\hat{s}(h)}, h \in Q\right\}$ are the singular values of $\Phi$;

b) $\{\hat{s}(h), h \in Q\}$ is the FT of the inner-product sequence $\{\langle\phi(0), \phi(q)\rangle, q \in Q\}$;

c) $\mathcal{I}$ is the set of indexes $h \in Q$ for which $\sigma(h) \neq 0$;

d) $u(h)=\hat{\phi}(h) / \sigma(h)$ for $h \in \mathcal{I}$;

e) $\{\hat{\phi}(h), h \in Q\}$ is the FT of $\{\phi(q), q \in Q\}$;

2) the canonical tight frame vectors $\left\{\mu_{i}, 1 \leq i \leq n\right\}$ are GU with generating group $\mathcal{Q}$ and generating vector $\mu=$ $(1 / \sqrt{n}) \sum_{h \in \mathcal{I}} u(h)$

3) the frame bounds of the frame $\left\{\phi_{i}, 1 \leq i \leq n\right\}$ are given by $A=\sqrt{n} \min _{h \in \mathcal{I}} \hat{s}(h)$ and $B=\sqrt{n} \max _{h \in \mathcal{I}} \hat{s}(h)$.

An important special case of Theorem 2 is the case in which the generating group $\mathcal{Q}$ is cyclic so that $U_{i}=V^{i-1}, 1 \leq i \leq n$, where $V$ is a unitary matrix with $V^{n}=I$. A cyclic group generates a cyclic vector set $\mathcal{S}=\left\{\phi_{i}=V^{i-1} \phi, 1 \leq i \leq n\right\}$, where $\phi$ is arbitrary. For example, the frame in Fig. 1 is cyclic with $V$ denoting a rotation by $120^{\circ}$. If $\mathcal{Q}$ is cyclic, then $G$ is a circulant matrix, ${ }^{5}$ and $Q$ is the cyclic group $\mathbb{Z}_{n}$. The FT kernel is then $\langle h, g\rangle=e^{-2 \pi i h g / n}$ for $h, g \in \mathbb{Z}_{n}$, and the FT matrix $\mathcal{F}$ reduces to the $n \times n$ discrete FT (DFT) matrix. The singular values of $\Phi$ are then $n^{1 / 4}$ times the square roots of the DFT values of the inner products $\left\{\left\langle\phi_{1}, \phi_{j}\right\rangle, 1 \leq j \leq n\right\}$.

\section{B. Properties of the Canonical Tight Frame}

The canonical tight-frame vectors $\mu_{i}$ corresponding to the frame-vectors $\phi_{i}$ have the property that they are the closest normalized tight-frame vectors to the vectors $\phi_{i}$, in a least-squares

\footnotetext{
${ }^{5} \mathrm{~A}$ circulant matrix is a matrix where every row (or column) is obtained by a right circular shift (by one position) of the previous row (or column). An example is
}

$$
\left[\begin{array}{lll}
a_{0} & a_{2} & a_{1} \\
a_{1} & a_{0} & a_{2} \\
a_{2} & a_{1} & a_{0}
\end{array}\right] .
$$

sense [29], [27], [30]. Thus, the vectors $\mu_{i}$ are the normalized tight-frame vectors that minimize the least-squares error

$$
\sum_{i=1}^{n}\left\langle\phi_{i}-\mu_{i}, \phi_{i}-\mu_{i}\right\rangle
$$

We now show that when the original frame vectors $\phi_{i}$ are GU with generating group $\mathcal{Q}$, the canonical tight-frame vectors have the additional property that among all normalized tight-frame vectors they maximize

$$
R_{\phi \mu}=\sum_{i=1}^{n}\left|\left\langle\phi_{i}, \mu_{i}\right\rangle\right|^{2}
$$

Maximizing $R_{\phi \mu}$ may be of interest in various applications. For example, in a matched-filter detection problem considered in [12], $R_{\phi \mu}$ represents the total output signal-to-noise ratio. As another example, in a multiuser detection problem considered in [13], maximizing $R_{\phi \mu}$ has the effect of minimizing the multipleaccess interference at the input of the proposed detector.

To obtain a more convenient expression for $R_{\phi \mu}$, let $\Phi$ and $M$ denote the matrices of columns $\phi_{i}$ and $\mu_{i}$, respectively. Since the vectors $\mu_{i}$ form a normalized tight frame for $\mathcal{H}, M$ satisfies

$$
M M^{*}=I_{m} \text {. }
$$

From Corollary 1, $\Phi$ has an SVD of the form $\Phi=U \Sigma \mathcal{F}^{*}$, where $U$ is unitary, $\mathcal{F}$ is the FT matrix over the additive group $Q$ to which $\mathcal{Q}$ is isomorphic, and $\Sigma$ is an $m \times n$ diagonal matrix with diagonal elements $\sigma_{i}>0$. From (32), it follows that $M$ can be written as $M=U \tilde{I} Z^{*}$ where $Z$ is an arbitrary unitary matrix and $\tilde{I}$ is an $m \times n$ diagonal matrix with diagonal elements all equal to 1 .

Let $f_{i}$ and $z_{i}$ denote the columns of $\mathcal{F}^{*}$ and $Z^{*}$, respectively. Then we can express $R_{\phi \mu}$ as

$$
\begin{aligned}
R_{\phi \mu} & =\sum_{i=1}^{n}\left|\left\langle\phi_{i}, \mu_{i}\right\rangle\right|^{2}=\sum_{i=1}^{n}\left|\left\langle U^{*} \phi_{i}, U^{*} \mu_{i}\right\rangle\right|^{2} \\
& =\sum_{i=1}^{n}\left|\left\langle f_{i}, \bar{\Sigma} z_{i}\right\rangle\right|^{2}
\end{aligned}
$$

where $\bar{\Sigma}$ is an $n \times n$ diagonal matrix with the first $m$ diagonal elements equal to $\sigma_{i}$, and the remaining diagonal elements are all equal to 0 .

Our problem then reduces to finding a set of orthonormal vectors $z_{i}$ that maximize $\sum_{i}\left|\left\langle f_{i}, \bar{\Sigma} z_{i}\right\rangle\right|^{2}$, where the vectors $f_{i}$ are also orthonormal. Using the Cauchy-Schwarz inequality, we have that

$$
R_{\phi \mu}=\sum_{i=1}^{n}\left|\left\langle\bar{\Sigma}^{1 / 2} f_{i}, \bar{\Sigma}^{1 / 2} z_{i}\right\rangle\right|^{2} \leq \sum_{i=1}^{n}\left\langle f_{i}, \bar{\Sigma} f_{i}\right\rangle\left\langle z_{i}, \bar{\Sigma} z_{i}\right\rangle
$$

with equality if and only if $\bar{\Sigma}^{1 / 2} f_{i}=c_{i} \bar{\Sigma}^{1 / 2} z_{i}$ for some $c_{i}$. In particular, we have equality for $z_{i}=f_{i}$. Since the components of the vectors $f_{i}$ all have equal magnitude $1 / \sqrt{n}$,

$$
\left\langle f_{i}, \bar{\Sigma} f_{i}\right\rangle=\frac{1}{n} \sum_{k=1}^{m} \sigma_{k} \triangleq \alpha, \quad \text { for all } i
$$






Fig. 2. Example of a GU frame.

and (34) reduces to

$$
R_{\phi \mu} \leq \alpha \sum_{i=1}^{n}\left\langle z_{i}, \bar{\Sigma} z_{i}\right\rangle=\alpha \operatorname{Tr}\left(Z^{*} \bar{\Sigma} Z\right)=\alpha \operatorname{Tr}(\bar{\Sigma})=n \alpha^{2}
$$

with equality if $f_{i}=z_{i}$.

The normalized tight-frame vectors that maximize $R_{\phi \mu}$ are then the columns of $\hat{M}=U \tilde{I} \mathcal{F}^{*}=S^{-1 / 2} \Phi$ where $S=\Phi \Phi^{*}$, and are equal to the canonical tight frame vectors.

\section{EXAMPLE OF A GU FRAME}

We now consider an example demonstrating the ideas of the previous section.

Consider the frame vectors $\phi_{1}=1 / 2\left[\begin{array}{ll}\sqrt{3} & -1\end{array}\right]^{*}, \phi_{2}=$ $1 / 2\left[\begin{array}{ll}\sqrt{3} & 1\end{array}\right]^{*}, \phi_{3}=1 / 2\left[\begin{array}{ll}-\sqrt{3} & 1\end{array}\right]^{*}, \phi_{4}=1 / 2\left[\begin{array}{ll}-\sqrt{3} & -1\end{array}\right]^{*}$, depicted in Fig. 2.

The corresponding Gram matrix is given by

$$
G=\left[\begin{array}{cccc}
1 & 0.5 & -1 & -0.5 \\
0.5 & 1 & -0.5 & -1 \\
-1 & -0.5 & 1 & 0.5 \\
-0.5 & -1 & 0.5 & 1
\end{array}\right]
$$

which is a permuted matrix with $G=G^{T}$. From Proposition 2 it follows that the vectors $\phi_{i}$ are GU. Since the vectors $\phi_{i}$ also span $\mathbb{R}^{2}$, these vectors form a GU frame for $\mathbb{R}^{2}$.

The vectors $\phi_{i}$ can be expressed as $\left\{\phi_{i}=U_{i} \phi, 1 \leq i \leq 4\right\}$, where $\phi=\phi_{1}$ and the matrices $\left\{U_{i}, 1 \leq i \leq 4\right\}$ are unitary, form an Abelian group $\mathcal{Q}$, and are given by

$$
\begin{array}{rlrl}
U_{1} & =I_{2}, & U_{2} & =\left[\begin{array}{rr}
1 & 0 \\
0 & -1
\end{array}\right] \\
U_{3}=\left[\begin{array}{rr}
-1 & 0 \\
0 & -1
\end{array}\right], & U_{4}=\left[\begin{array}{rr}
-1 & 0 \\
0 & 1
\end{array}\right] .
\end{array}
$$

The multiplication table of the group $\mathcal{Q}$ is

\begin{tabular}{c|cccc} 
& $U_{1}$ & $U_{2}$ & $U_{3}$ & $U_{4}$ \\
\hline$U_{1}$ & $U_{1}$ & $U_{2}$ & $U_{3}$ & $U_{4}$ \\
$U_{2}$ & $U_{2}$ & $U_{1}$ & $U_{4}$ & $U_{3}$ \\
$U_{3}$ & $U_{3}$ & $U_{4}$ & $U_{1}$ & $U_{2}$ \\
$U_{4}$ & $U_{4}$ & $U_{3}$ & $U_{2}$ & $U_{1}$.
\end{tabular}

If we define the correspondence

$$
U_{1} \leftrightarrow(0,0), \quad U_{2} \leftrightarrow(0,1), \quad U_{3} \leftrightarrow(1,0), \quad U_{4} \leftrightarrow(1,1)
$$

then this table becomes the addition table of $Q=\mathbb{Z}_{2} \times \mathbb{Z}_{2}$

\begin{tabular}{l|llll} 
& $(0,0)$ & $(0,1)$ & $(1,0)$ & $(1,1)$ \\
\hline$(0,0)$ & $(0,0)$ & $(0,1)$ & $(1,0)$ & $(1,1)$ \\
$(0,1)$ & $(0,1)$ & $(0,0)$ & $(1,1)$ & $(1,0)$ \\
$(1,0)$ & $(1,0)$ & $(1,1)$ & $(0,0)$ & $(0,1)$ \\
$(1,1)$ & $(1,1)$ & $(1,0)$ & $(0,1)$ & $(0,0)$.
\end{tabular}

Only the way in which the elements are labeled distinguishes the table of (40) from the table of (38); thus, $\mathcal{Q}$ is isomorphic to $Q$. Over $Q=\mathbb{Z}_{2} \times \mathbb{Z}_{2}$, the FT matrix $\mathcal{F}$ is the Hadamard matrix

$$
\mathcal{F}=\frac{1}{2}\left[\begin{array}{rrrr}
1 & 1 & 1 & 1 \\
1 & -1 & 1 & -1 \\
1 & 1 & -1 & -1 \\
1 & -1 & -1 & 1
\end{array}\right]
$$

From Theorem 2, the dual-frame vectors and the canonical tight-frame vectors are also GU with generators $\bar{\phi}$ and $\mu$, respectively, whose expressions are given in the theorem. Thus, to compute the dual and canonical tight-frame vectors we compute these generators and then apply the group $\mathcal{Q}$.

We first determine the FT of the first row of $G$ denoted by $s$

$$
\hat{s}=\mathcal{F} s=\frac{1}{2}\left[\begin{array}{llll}
0 & 0 & 3 & 1
\end{array}\right]^{*} .
$$

Using Theorem 2, it follows from (42) that the frame bounds are given by $A=1$ and $B=3$. Next, we compute the vectors $\hat{\phi}(h)$ which are the columns of

$$
\Phi \mathcal{F}=\left[\begin{array}{rrcr}
0 & 0 & 1.7 & 0 \\
0 & 0 & 0 & -1
\end{array}\right] .
$$

Using the expressions of the theorem, we then have that $\bar{\phi}=$ $[0.3-0.5]^{*}$ and $\mu=\left[\begin{array}{ll}0.5 & -0.5\end{array}\right]^{*}$. By applying the group $\mathcal{Q}$ to these generators we obtain that the dual-frame vectors are the columns of

$$
\bar{\Phi}=\left[\begin{array}{rrrr}
0.3 & 0.3 & -0.3 & -0.3 \\
-0.5 & 0.5 & 0.5 & -0.5
\end{array}\right]
$$

and the canonical tight-frame vectors are the columns of

$$
M=\left[\begin{array}{rrrr}
0.5 & 0.5 & -0.5 & -0.5 \\
-0.5 & 0.5 & 0.5 & -0.5
\end{array}\right] .
$$

Comparing (44) and (45) with the original frame vectors $\phi_{i}$, it is evident that the dual and canonical tight-frame vectors have the same symmetries as the original frame vectors, as illustrated in Fig. 3.

\section{COMPOUND GU FRAMES}

In Section IV, we showed that the dual and canonical tightframe vectors associated with a GU frame are themselves GU and can, therefore, be computed using a single generator. In this section, we consider a class of frames which consist of subsets that are $\mathrm{GU}$, and are, therefore, referred to as compound $G U$ $(C G U)$ frames. As we show, the dual and canonical tight-frame vectors associated with a CGU frame share the same symmetries as the original frame and can be computed using a set of generators.

A set of frame vectors $\left\{\phi_{i k}, 1 \leq i \leq l, 1 \leq k \leq r\right\}$ is CGU if $\phi_{i k}=U_{i} \phi_{k}$ for some generating vectors $\left\{\phi_{k}, 1 \leq k \leq r\right\}$, 




Fig. 3. Symmetry property of the frame vectors $\phi_{i}$, the dual-frame vectors $\overline{\phi_{i}}$, and the canonical tight-frame vectors $\mu_{i} . \bar{\phi}_{i}$ are the columns of $\bar{\Phi}$ given by (44), and $\mu_{i}$ are the columns of $M$ given by (45). The frame vectors, dual-frame vectors, and the canonical tight-frame vectors all have the same symmetry properties.

and the matrices $\left\{U_{i}, 1 \leq i \leq l\right\}$ are unitary and form an Abelian group $\mathcal{Q}$.

A CGU frame is in general not GU. However, for every $k$, the vectors $\left\{\phi_{i k}, 1 \leq i \leq l\right\}$ are a GU vector set with generating group $\mathcal{Q}$.

A special case of CGU frames are filter-bank frames studied for $L_{2}(\mathbb{R})$ in [7]-[9], in which $\mathcal{Q}$ is the group of translations by integer multiples of the subsampling factor, and the generating vectors are the filter-bank synthesis filters.

As we show in the following proposition, the frame bounds of a CGU frame can be bounded by the sum of the norms of the generating vectors.

Proposition 3: Let $\mathcal{S}=\left\{\phi_{i k}=U_{i} \phi_{k}, 1 \leq i \leq l, 1 \leq k \leq r\right\}$ be a compound GU frame with frame bounds $\bar{A}$ and $\bar{B}$, where $\left\{\phi_{k}, 1 \leq k \leq r\right\}$ is an arbitrary set of generating vectors. Then

$$
A \leq \frac{l}{m} \sum_{k=1}^{r}\left\|\phi_{k}\right\|^{2} \leq B .
$$

If, in addition, the frame is tight, then

$$
A=\frac{l}{m} \sum_{k=1}^{r}\left\|\phi_{k}\right\|^{2} .
$$

Proof: We can express the frame operator corresponding to the frame vectors $\phi_{i}$ as

$$
S=\sum_{i=1}^{l} \sum_{k=1}^{r} U_{i} \phi_{k} \phi_{k}^{*} U_{i}^{*} .
$$

Then

$$
\operatorname{Tr}(S)=\sum_{i=1}^{l} \sum_{k=1}^{r} \operatorname{Tr}\left(U_{i} \phi_{k} \phi_{k}^{*} U_{i}^{*}\right)=l \sum_{k=1}^{r}\left\|\phi_{k}\right\|^{2}
$$

so that

$$
\sum_{i=1}^{m} \lambda_{i}(S)=\operatorname{Tr}(S)=l \sum_{k=1}^{r}\left\|\phi_{k}\right\|^{2}
$$

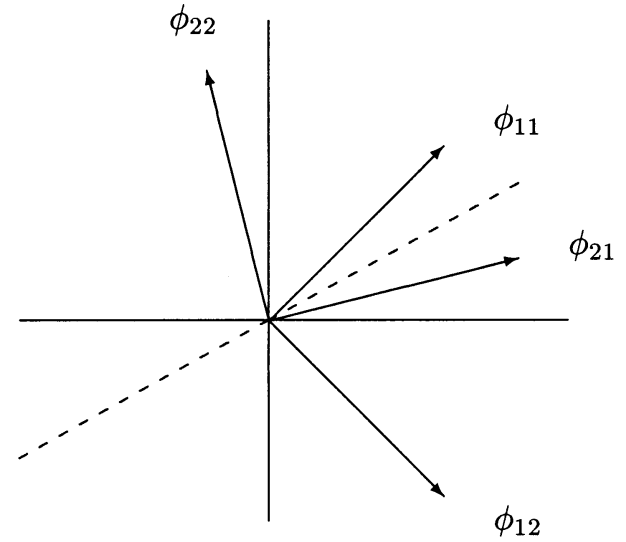

Fig. 4. A compound GU frame. The sets $\mathcal{S}_{1}=\left\{\phi_{11}, \phi_{21}\right\}$ and $\mathcal{S}_{2}=$ $\left\{\phi_{12}, \phi_{22}\right\}$ are both GU with the same generating group; both sets are invariant under a reflection about the dashed line. However, the combined set $\mathcal{S}=\left\{\phi_{11}, \phi_{21}, \phi_{12}, \phi_{22}\right\}$ is no longer GU.

Therefore,

$$
A=\min _{i} \lambda_{i}(S) \leq \frac{1}{m} \sum_{i=1}^{m} \lambda_{i}(S)=\frac{l}{m} \sum_{k=1}^{r}\left\|\phi_{k}\right\|^{2}
$$

and

$$
B=\max _{i} \lambda_{i}(S) \geq \frac{1}{m} \sum_{i=1}^{m} \lambda_{i}(S)=\frac{l}{m} \sum_{k=1}^{r}\left\|\phi_{k}\right\|^{2} .
$$

\section{A. Example of a $C G U$ Frame}

An example of a CGU frame is illustrated in Fig. 4. In this example, the frame vectors are $\left\{\phi_{i k}, 1 \leq i, k \leq 2\right\}$ where $\left\{\phi_{i k}=U_{i} \phi_{k}, U_{i} \in \mathcal{Q}\right\}, \mathcal{Q}=\left\{I_{2}, U\right\}$ with

$$
U=\frac{1}{2}\left[\begin{array}{cc}
1 & \sqrt{3} \\
\sqrt{3} & -1
\end{array}\right]
$$

and the generating vectors are

$$
\phi_{1}=\frac{1}{\sqrt{2}}\left[\begin{array}{l}
1 \\
1
\end{array}\right], \quad \phi_{2}=\frac{1}{\sqrt{2}}\left[\begin{array}{r}
1 \\
-1
\end{array}\right] .
$$

The matrix $U$ represents a reflection about the dashed line in Fig. 4. Thus, the vector $\phi_{21}$ is obtained by reflecting the generator $\phi_{11}$ about this line, and similarly, the vector $\phi_{22}$ is obtained by reflecting the generator $\phi_{12}$ about this line.

As can be seen from the figure, the frame is not GU. In particular, there is no isometry that transforms $\phi_{11}$ into $\phi_{12}$ while leaving the set invariant. However, the sets $\mathcal{S}_{1}=\left\{\phi_{11}, \phi_{21}\right\}$ and $\mathcal{S}_{2}=\left\{\phi_{12}, \phi_{22}\right\}$ are both GU with generating group $\mathcal{Q}$.

\section{B. Dual and Canonical Tight Frames Associated With CGU Frames}

We now show that the dual and canonical tight frames associated with a CGU frame are also CGU.

Expressing the frame operator as

$$
S=\sum_{i=1}^{l} \sum_{k=1}^{r} \phi_{i k} \phi_{i k}^{*}=\sum_{i=1}^{l} U_{i}\left(\sum_{k=1}^{r} \phi_{k} \phi_{k}^{*}\right) U_{i}^{*}
$$


for all $j$ we have that

$$
\begin{aligned}
S U_{j} & =\sum_{i=1}^{l} U_{i}\left(\sum_{k=1}^{r} \phi_{k} \phi_{k}^{*}\right) U_{i}^{*} U_{j} \\
& =U_{j} \sum_{i=1}^{l} U_{j}^{*} U_{i}\left(\sum_{k=1}^{r} \phi_{k} \phi_{k}^{*}\right) U_{i}^{*} U_{j} \\
& =U_{j} \sum_{i=1}^{l} U_{i}\left(\sum_{k=1}^{r} \phi_{k} \phi_{k}^{*}\right) U_{i}^{*}=U_{j} S
\end{aligned}
$$

since $\left\{U_{j}^{*} U_{i}, 1 \leq i \leq l\right\}$ is just a permutation of $\mathcal{Q}$. Thus, $S$ commutes with $U_{j}$, so that $S^{-1}$ and $S^{-1 / 2}$ also commute with $U_{j}$ for all $j$. Then, the dual-frame vectors $\bar{\phi}_{i k}$ of the vectors $\phi_{i k}$ are given by

$$
\bar{\phi}_{i k}=S^{-1} \phi_{i k}=S^{-1} U_{i} \phi_{k}=U_{i} S^{-1} \phi_{k}=U_{i} \bar{\phi}_{k}
$$

where $\bar{\phi}_{k}=S^{-1} \phi_{k}$, which shows that the dual-frame vectors $\left\{\bar{\phi}_{i k}=S^{-1} \phi_{i k}\right\}$ are CGU with generating group equal to $\mathcal{Q}$.

Similarly

$$
\mu_{i k}=S^{-1 / 2} \phi_{i k}=S^{-1 / 2} U_{i} \phi_{k}=U_{i} S^{-1 / 2} \phi_{k}=U_{i} \mu_{k}
$$

where $\mu_{k}=S^{-1 / 2} \phi_{k}$, which shows that the canonical tightframe vectors $\left\{\mu_{i k}=S^{-1 / 2} \phi_{i k}\right\}$ are also CGU with generating group $\mathcal{Q}$.

Therefore, to compute the dual-frame vectors or the canonical tight-frame vectors all we need is to compute the generating vectors $\left\{\bar{\phi}_{k}, 1 \leq k \leq r\right\}$ and $\left\{\mu_{k}, 1 \leq k \leq r\right\}$, respectively. The remaining frame vectors are then obtained by applying the group $\mathcal{Q}$ to the corresponding set of generating vectors.

For the CGU set of Fig. 4 we have that

$$
\Phi \Phi^{*}=2\left[\begin{array}{ll}
1 & 0 \\
0 & 1
\end{array}\right] .
$$

Therefore, $\left\{\bar{\phi}_{k}=(1 / 2) \phi_{k}, 1 \leq k \leq r\right\}$ and $\left\{\mu_{k}=(1 / \sqrt{2}) \phi_{k}\right.$, $1 \leq k \leq r\}$. Since in this example the generating vectors of the dual frame and the canonical frame are proportional to the generating vectors of the original frame, the dual-frame vectors and the canonical frame vectors are proportional to the original frame vectors.

\section{CGU Frames With GU Generators}

A special class of CGU frames is $C G U$ frames with $G U$ generators in which the generating vectors $\left\{\phi_{k}, 1 \leq k \leq r\right\}$ are themselves GU. Specifically, $\left\{\phi_{k}=V_{k} \phi\right\}$ for some generator $\phi$, where the matrices $\left\{V_{k}, 1 \leq k \leq r\right\}$ are unitary, and form an Abelian group $\mathcal{G}$.

Now suppose that $U_{p}$ and $V_{t}$ commute up to a phase factor for all $t$ and $p$ so that $U_{p} V_{t}=V_{t} U_{p} e^{j \theta(p, t)}$ where $\theta(p, t)$ is an arbitrary phase function that may depend on the indexes $p$ and $t$. In this case, we say that $\mathcal{Q}$ and $\mathcal{G}$ commute up to a phase factor. Then for all $p, t$

$$
\begin{aligned}
S U_{p} V_{t} & =\sum_{i=1}^{l} U_{i}\left(\sum_{k=1}^{r} V_{k} \phi \phi^{*} V_{k}^{*}\right) U_{i}^{*} U_{p} V_{t} \\
& =U_{p} V_{t} \sum_{i=1}^{l} V_{t}^{*} U_{p}^{*} U_{i}\left(\sum_{k=1}^{r} V_{k} \phi \phi^{*} V_{k}^{*}\right) U_{i}^{*} U_{p} V_{t}
\end{aligned}
$$

$$
\begin{aligned}
& =U_{p} V_{t} \sum_{i=1}^{l} V_{t}^{*} U_{i}^{*}\left(\sum_{k=1}^{r} V_{k} \phi \phi^{*} V_{k}^{*}\right) U_{i} V_{t} \\
& =U_{p} V_{t} \sum_{i=1}^{l} U_{i}^{*}\left(\sum_{k=1}^{r} V_{t}^{*} V_{k} \phi \phi^{*} V_{k}^{*} V_{t}\right) U_{i} \\
& =U_{p} V_{t} \sum_{i=1}^{l} U_{i}\left(\sum_{k=1}^{r} V_{k} \phi \phi^{*} V_{k}^{*}\right) U_{i}^{*}=U_{p} V_{t} S .
\end{aligned}
$$

The dual frame vectors $\bar{\phi}_{i k}$ of the vectors $\phi_{i k}$ are then given by

$$
\bar{\phi}_{i k}=S^{-1} \phi_{i k}=S^{-1} U_{i} V_{k} \phi=U_{i} V_{k} S^{-1} \phi=U_{i} V_{k} \bar{\phi}
$$

where $\bar{\phi}=S^{-1} \phi$. Similarly

$$
\mu_{i k}=S^{-1 / 2} \phi_{i k}=S^{-1 / 2} U_{i} V_{k} \phi=U_{i} V_{k} S^{-1 / 2} \phi=U_{i} V_{k} \mu
$$

where $\mu=S^{-1 / 2} \phi$. Thus, even though the frame is not in general GU, the dual and canonical tight-frame vectors can be computed using a single generating vector.

Alternatively, we can express $\bar{\phi}_{i k}$ as $\bar{\phi}_{i k}=U_{i} \bar{\phi}_{k}$ where $\bar{\phi}_{k}=$ $V_{k} \bar{\phi}$. Similarly, $\mu_{i k}=U_{i} \mu_{k}$ where $\mu_{k}=V_{k} \bar{\mu}$. It then follows that the generators $\bar{\phi}_{k}$ and $\mu_{k}$ are both GU with generating group $\mathcal{G}=\left\{V_{k}, 1 \leq k \leq r\right\}$.

We conclude that for a CGU frame with commuting GU generators and generating group $\mathcal{Q}$, the dual frame and the canonical frame are also CGU with commuting GU generators and generating group $\mathcal{Q}$.

As we now show, in the special case in which $\theta=0$ so that $U_{i} V_{k}=V_{k} U_{i}$ for all $i, k$, the resulting frame is GU. To this end, we need to show that the unitary matrices $\mathcal{Q}^{\prime}=$ $\left\{Q_{i k}=U_{i} V_{k}\right\}$ form an Abelian group. First,

$$
Q_{i k} Q_{j t}=U_{i} V_{k} U_{j} V_{t}=U_{i} U_{j} V_{k} V_{t} .
$$

Since $U_{i} U_{j} \in \mathcal{Q}$ and $V_{k} V_{t} \in \mathcal{G}, Q_{i k} Q_{j t} \in \mathcal{Q}^{\prime}$. Next,

$$
Q_{i k}^{-1}=V_{k}^{*} U_{i}^{*}=U_{i}^{*} V_{k}^{*} \in \mathcal{Q}^{\prime}
$$

since $U_{i}^{*} \in \mathcal{Q}$ and $V_{k}^{*} \in \mathcal{G}$. Also, $I \in \mathcal{Q}^{\prime}$ since $I \in \mathcal{Q}$ and $I \in \mathcal{G}$. Finally,

$Q_{i k} Q_{j t}=U_{i} V_{k} U_{j} V_{t}=U_{i} U_{j} V_{t} V_{k}=U_{j} V_{t} U_{i} V_{k}=Q_{j t} Q_{i k}$

A special case of $\mathrm{CGU}$ frames with $\mathrm{GU}$ generators for which $\mathcal{Q}$ and $\mathcal{G}$ commute up to a phase factor are the WH frames [17], [3], [16]. If the WH frame is critically sampled, then $\theta(p, t)=0$ and the $\mathrm{WH}$ frame reduces to a GU frame. In the more general oversampled case, $\theta(p, t) \neq 0$.

To summarize, we have the following theorem.

Theorem 3 (CGU Frames): Let

$$
\mathcal{S}=\left\{\phi_{i k}=U_{i} \phi_{k}, 1 \leq i \leq l, 1 \leq k \leq r\right\}
$$

be a CGU frame with generating vectors $\left\{\phi_{k}, 1 \leq k \leq r\right\}$ and generating group $\mathcal{Q}$, and let $S$ be the frame operator corresponding to the frame vectors $\left\{\phi_{i k}\right\}$. Then

1) the dual frame vectors $\left\{\bar{\phi}_{i k}, 1 \leq i \leq l, 1 \leq k \leq r\right\}$ are CGU with generating group $\mathcal{Q}$ and generating vectors $\left\{\bar{\phi}_{k}=S^{-1} \phi_{k}, 1 \leq k \leq r\right\}$; 
2) the canonical tight-frame vectors $\left\{\mu_{i k}, 1 \leq i \leq l, 1 \leq\right.$ $k \leq r\}$ are $\mathrm{CGU}$ with generating group $\mathcal{Q}$ and generating vectors $\left\{\mu_{k}=S^{-1 / 2} \phi_{k}, 1 \leq k \leq r\right\}$.

If in addition, the generating vectors $\left\{\phi_{k}=V_{k} \phi, 1 \leq k \leq r\right\}$ are GU with $U_{i} V_{k}=V_{k} U_{i} e^{j \theta(i, k)}$ for all $i, k$, then

1) $\bar{\phi}_{i k}=U_{i} V_{k} \bar{\phi}$ where $\bar{\phi}=S^{-1} \phi$ so that the dual-frame vectors are $\mathrm{CGU}$ with $\mathrm{GU}$ generators;

2) $\mu_{i k}=U_{i} V_{k} \mu$ where $\mu=S^{-1 / 2} \phi$ so that the canonical frame vectors are CGU with GU generators;

$3)$ if in addition $\theta(i, k)=0$ for all $i, k$, then the vectors $\left\{\phi_{i k}, 1 \leq i \leq l, 1 \leq k \leq r\right\}$ form a GU frame.

\section{PRUNING GU FRAMES}

In applications, it is often desirable to know how a frame behaves when one or more frame elements are removed. In particular, it is important to know or to be able to estimate the frame bounds of the reduced frame. In general, if no structural constraints are imposed on a frame, this behavior will depend critically on the particular frame elements removed. For example, removal of a particular frame element may destroy the frame property so that the remaining vectors do not constitute a frame anymore, whereas if a different element is removed the remaining vectors may still constitute a frame.

One of the prime applications of frames is signal analysis and synthesis, where a signal is expanded by computing the inner products of the signal with the frame elements. The resulting coefficients are subsequently stored, transmitted, quantized, or manipulated in some way. In particular, a coefficient may be lost (e.g., due to a transmission error) which results in a reconstructed signal that is equivalent to an expansion using a pruned frame obtained by removing the corresponding frame vector.

Recently, there has been increased interest in using frames for multiple-description source coding where a signal is expanded into a redundant set of functions and the resulting coefficients are transmitted over a lossy packet network, where one or more of the coefficients can be lost because a packet is dropped [23], [14]. The goal of multiple description source coding is to ensure a gradually behaving reconstruction quality as a function of the number of dropped packets. When using frames in this context, the reconstruction quality is often governed by the frame bound ratio of the pruned frame. If the packets are dropped with equal probability, then it is desirable that the frame bound ratio should deteriorate uniformly irrespectively of the particular frame element that is removed. In the following, we show that GU frames have this property. We, furthermore, demonstrate that if the original frame is a tight GU frame, then the frame bound ratio of the pruned frame obtained by removing one frame element can be computed exactly. We also consider the case where sets of frame elements are removed.

Theorem 4 (Pruned GU Frames): Let

$$
\mathcal{S}=\left\{\phi_{i}=U_{i} \phi, U_{i} \in \mathcal{Q}\right\}
$$

be a GU frame generated by a finite Abelian group $\mathcal{Q}$ of unitary matrices, where $\phi$ is an arbitrary generating vector. Let $\Phi$ be the matrix of columns $\phi_{i}$, and let $S=\Phi \Phi^{*}$ be the corresponding frame operator. Let

$$
\mathcal{S}(j)=\left\{\phi_{i}=U_{i} \phi, U_{i} \in \mathcal{Q}, i \neq j\right\}
$$

be the pruned set obtained by removing the element $\phi_{j}$. Then the eigenvalues of the frame operator corresponding to the pruned set do not depend on the particular element $\phi_{j}$ removed.

Proof: The frame operator corresponding to the pruned frame is given by

$$
S(j)=\sum_{i=1}^{n} U_{i} \phi \phi^{*} U_{i}^{*}-U_{j} \phi \phi^{*} U_{j}^{*} .
$$

Since $U_{j}$ is unitary, the eigenvalues of $S(j)$ are equal to the eigenvalues of $U_{j}^{*} S(j) U_{j}$. But

$$
\begin{aligned}
U_{j}^{*} S(j) U_{j} & =U_{j}^{*} \sum_{i=1}^{n} U_{i} \phi \phi^{*} U_{i}^{*} U_{j}-\phi \phi^{*} \\
& =\sum_{i=1}^{n} U_{i} \phi \phi^{*} U_{i}^{*}-\phi \phi^{*}=S-\phi \phi^{*} .
\end{aligned}
$$

Since $U_{j}^{*} S(j) U_{j}$ is independent of $j$, the eigenvalues of $S(j)$ do not depend on $j$.

In general, it is difficult to provide estimates on the frame bounds of the pruned frame. However, in the special case where the original GU frame is a tight frame, these bounds can be determined exactly.

Corollary 2 (Pruned Tight GU Frames): Let

$$
\mathcal{S}=\left\{\phi_{i}=U_{i} \phi, U_{i} \in \mathcal{Q}\right\}
$$

be a GU tight frame generated by a finite Abelian group $\mathcal{Q}$ of unitary matrices, where $\phi$ is a unit norm generating vector. Let $\Phi$ be the matrix of columns $\phi_{i}$, and let $S=\Phi \Phi^{*}$ be the corresponding frame operator. Let

$$
\mathcal{S}(j)=\left\{\phi_{i}=U_{i} \phi, U_{i} \in \mathcal{Q}, i \neq j\right\}
$$

be the pruned set obtained by removing the element $\phi_{j}$. Then, the eigenvalues of the frame operator corresponding to the pruned set are given by $\lambda_{1}=\frac{n}{m}-1$ and $\lambda_{i}=\frac{n}{m}, 2 \leq i \leq n$, independent of $\phi_{j}$.

A similar result was also shown by Goyal et al. [14, Theorem 4.1].

Proof: Since $\mathcal{S}$ is a tight frame with $\|\phi\|=1$, from Proposition 1, the frame bound $A=n / m$ and $S=(n / m) I_{m}$. Then

$$
S(j)=\frac{n}{m} I_{m}-U_{j} \phi \phi^{*} U_{j}^{*}
$$

and

$$
U_{j}^{*} S(j) U_{j}=\frac{n}{m} I_{m}-\phi \phi^{*} .
$$

Since $\|\phi\|=1, \phi \phi^{*}$ has one eigenvalue equal to 1 , and the remaining eigenvalues equal to 0 . The eigenvalues of $S(j)$ are therefore given by $\lambda_{1}=\frac{n}{m}-1$ and $\lambda_{i}=\frac{n}{m}, 2 \leq i \leq n$.

An immediate consequence of Corollary 2 is that the frame bound ratio of the pruned frame is given by $B / A=1 /(1-$ $m / n)$, which is close to 1 for large redundancy $r=n / m$.

We next consider the case where multiple frame elements are removed. 
Corollary 3: Let $\mathcal{S}=\left\{\phi_{i}=U_{i} \phi, U_{i} \in \mathcal{Q}\right\}$ be a GU frame generated by a finite Abelian group $\mathcal{Q}$ of unitary matrices, let $\Phi$ be the matrix of columns $\phi_{i}$, and let $S=\Phi \Phi^{*}$ be the corresponding frame operator. Let $\mathcal{J}$ be a set of indexes, and let $\mathcal{J}(k)$ denote the set of indexes $i$ such that $U_{i}=U_{k} U_{j}$ for fixed $k$ and $j \in \mathcal{J}$. Let $\mathcal{S}(k)=\left\{\phi_{i}=U_{i} \phi, U_{i} \in \mathcal{Q}, i \neq \mathcal{J}(k)\right\}$ be a pruned set obtained by removing the elements $\phi_{i}$ with $i \in \mathcal{J}(k)$. Then, the eigenvalues of the frame operator corresponding to the pruned set are independent of $k$.

Proof: The frame operator corresponding to the pruned frame is given by

$$
S(k)=\sum_{i=1}^{n} U_{i} \phi \phi^{*} U_{i}^{*}-U_{k}\left[\sum_{j \in \mathcal{J}} U_{j} \phi \phi^{*} U_{j}^{*}\right] U_{k}^{*} .
$$

Then

$$
\begin{aligned}
U_{k}^{*} S(k) U_{k} & =U_{k}^{*} \sum_{i=1}^{n} U_{i} \phi \phi^{*} U_{i}^{*} U_{k}-\sum_{j \in \mathcal{J}} U_{j} \phi \phi^{*} U_{j}^{*} \\
& =\sum_{i=1}^{n} U_{i} \phi \phi^{*} U_{i}^{*}-\sum_{j \in \mathcal{J}} U_{j} \phi \phi^{*} U_{j}^{*}
\end{aligned}
$$

is independent of $k$, and, consequently, the eigenvalues of $S(k)$ do not depend on $k$.

To conclude this section, GU frames have strong symmetry properties in the sense that removing any one of the elements leads to a vector set with bounds independent of the particular element removed. Moreover, if the original frame is tight, then we can compute the bounds of the pruned frame exactly.

\section{CONSTRUCTING GU FRAMES}

Suppose we are given a set of vectors $\left\{\varphi_{i}, 1 \leq i \leq n\right\}$ that form a frame for an $m$-dimensional space $\mathcal{H}$. We would like to construct a GU frame $\left\{\phi_{i}, 1 \leq i \leq n\right\}$ from the vectors $\left\{\varphi_{i}\right\}$.

From Theorem 1, it follows that the vectors $\left\{\phi_{i}\right\}$ form a GU frame if and only if the Gram matrix $G$ has rank $m$, and is diagonalized by an FT matrix $\mathcal{F}$ over a finite product of cyclic groups. There are many ways to construct a frame from a given set of frame vectors $\varphi_{i}$ that satisfy these properties. For example, let $F$ be the matrix of columns $\varphi_{i}$, and let $F$ have an SVD $F=Q \Lambda V^{*}$, where $\Lambda$ is a diagonal matrix with diagonal elements $\lambda_{i}$. Then, the columns of $\Phi=F V \Sigma \mathcal{F}^{*}=Q \Lambda \Sigma \mathcal{F}^{*}$ form a GU frame, where $\mathcal{F}$ is any FT matrix over a product of cyclic groups, and $\Sigma$ is an arbitrary diagonal matrix with diagonal elements $\sigma_{i}>0$. The frame bounds of the resulting GU frame are given by $A=\min _{i} \lambda_{i}^{2} \sigma_{i}^{2}$ and $B=\max _{i} \lambda_{i}^{2} \sigma_{i}^{2}$, so that we can choose the diagonal matrix $\Sigma$ to control these bounds. In particular, choosing $\Sigma=I$ we have that the columns of $\Phi=F V \mathcal{F}^{*}=Q \Lambda \mathcal{F}^{*}$ form a GU frame. This choice has the property that the frame bounds of the GU frame are equal to the frame bounds of the original frame.

We now consider the problem of constructing an optimal GU frame. Specifically, let $\left\{\varphi_{i}, 1 \leq i \leq n\right\}$ denote a frame for $\mathcal{H}$, and suppose we wish to construct a GU frame $\left\{\phi_{i}\right\}$ from the vectors $\left\{\varphi_{i}\right\}$. A reasonable approach is to find a set of vectors $\phi_{i}$ that span $\mathcal{H}$, and are "closest" to the vectors $\varphi_{i}$ in the least- squares sense. Thus, we seek vectors $\phi_{i}$ that minimize the leastsquares error $E$, defined by

$$
E=\sum_{i=1}^{n}\left\langle e_{i}, e_{i}\right\rangle
$$

where $e_{i}$ denotes the $i$ th error vector

$$
e_{i}=\varphi_{i}-\phi_{i}
$$

subject to the constraint that the vectors $\phi_{i}$ form a GU frame.

If the vectors $\phi_{i}$ are GU, then their Gram matrix $G=\Phi^{*} \Phi$ is a permuted matrix with rank $m$, diagonalized by an FT matrix $\mathcal{F}$. Thus, the inner products $\left\{\left\langle\phi_{i}, \phi_{j}\right\rangle\right\}$ must satisfy

$$
\left\{\left\langle\phi_{i}, \phi_{j}\right\rangle, 1 \leq j \leq n\right\}=\beta^{2} P_{i}\left\{a_{j}, 1 \leq j \leq n\right\}
$$

where $P_{i}\left\{a_{j}, 1 \leq j \leq n\right\}$ is a permutation of the numbers $\left\{a_{j}, 1 \leq j \leq n\right\}, \beta>0$ is a scaling factor, and the numbers $\left\{a_{j}, 1 \leq j \leq n\right\}$ are chosen such that the matrix $R$, whose $i$ th row is equal to $P_{i}\left\{a_{j}, 1 \leq j \leq n\right\}$, is Hermitian, nonnegative definite, and diagonalized by an FT matrix $\mathcal{F}$.

In our development of the optimal GU frame vectors we assume that the permutations $P_{i}$ in (68) are specified. Since these permutations determine the additive group $Q$ over which the FT matrix $\mathcal{F}$ is defined, we assume that $\mathcal{F}$ is specified. We then consider three different constraints on the vectors $\phi_{i}$. First, we consider the case in which both the numbers $\left\{a_{j}, 1 \leq j \leq n\right\}$ and the scaling $\beta$ in (68) are known. The GU frame minimizing the least-squares error $E$ of (66) and (67) subject to this constraint is derived in Section VIII-A, and is referred to as the SC-LSGUF. Next, we consider the case in which the numbers $\left\{a_{j}, 1 \leq j \leq n\right\}$ in (68) are known, and the scaling $\beta$ is chosen to minimize $E$. The resulting $\mathrm{GU}$ frame is referred to as the C-LSGUF, and is derived in Section VIII-B. Finally, in Section VIII-C, we consider the more general case in which both the numbers $\left\{a_{j}, 1 \leq j \leq n\right\}$ and the scaling $\beta$ in (68) are chosen to minimize $E$. The resulting $\mathrm{GU}$ frame is referred to as the least-squares GU frame (LSGUF).

\section{A. Scaled-Constrained Least-Squares GU Frame (SC-LSGUF)}

We first consider the case in which the numbers $\left\{a_{j}, 1 \leq j \leq\right.$ $n\}$ and the scaling $\beta$ in (68) are known. Thus, we seek the set of vectors $\left\{\phi_{i}\right\}$ that minimize the least-squares error $E$ of (66) and (67) subject to the constraint

$$
\Phi^{*} \Phi=\beta_{0}^{2} R=\beta_{0}^{2} \mathcal{F} D \mathcal{F}^{*}
$$

where $\Phi$ is the matrix of columns $\phi_{i}, \beta_{0}$ is a known scaling factor, and $R$ is the matrix whose $i$ th row is equal to $P_{i}\left\{a_{j}, 1 \leq\right.$ $j \leq n\}$, where the numbers $\left\{a_{j}, 1 \leq j \leq n\right\}$ are given such that $R$ is diagonalized by $\mathcal{F}$ and $D$ is a diagonal matrix with diagonal elements $\left\{\alpha_{j}=n^{1 / 2} \hat{a}_{j}, 1 \leq j \leq n\right\}$ where $\left\{\hat{a}_{j}, 1 \leq j \leq n\right\}$ is the FT of the sequence $\left\{a_{j}, 1 \leq j \leq n\right\}$. From (69), the frame bounds of the vectors $\left\{\phi_{i}\right\}$ are given by $A=\beta_{0}^{2} \min _{j} \alpha_{j}$ and $B=\beta_{0}^{2} \max _{j} \alpha_{j}$.

The optimal SC-LSGUF vectors $\hat{\phi}_{i}$ follow from [34], and are the columns of $\hat{\Phi}$ where

$$
\hat{\Phi}=\beta_{0} U V^{*} \Sigma \mathcal{F}^{*} .
$$


Here, $U$ and $V$ are the right-hand unitary matrix and left-hand unitary matrix, respectively, in the SVD of $F \mathcal{F} \Sigma^{*}, F$ is the matrix of columns $\varphi_{i}$, and $\Sigma$ is an $m \times n$ diagonal matrix with diagonal elements $\sqrt{\alpha_{i}}$ for values of $i$ for which $\alpha_{i} \neq 0$.

If $F R F^{*}=\beta_{0}^{2} F \mathcal{F} D \mathcal{F}^{*} F$ is invertible, then we may express $\hat{\Phi}$ as

$$
\hat{\Phi}=\beta_{0}\left(F \mathcal{F} D \mathcal{F}^{*} F^{*}\right)^{-1 / 2} F \mathcal{F} D \mathcal{F}^{*}=\beta_{0}\left(F R F^{*}\right)^{-1 / 2} F R .
$$

\section{B. Constrained Least-Squares GU Frame (C-LSGUF)}

We now consider the case in which the numbers $\left\{a_{j}, 1 \leq\right.$ $j \leq n\}$ are known, but the scaling $\beta$ is not specified. Thus, we seek a set of vectors $\left\{\phi_{i}\right\}$ that minimize the least-squares error $E$ subject to

$$
\Phi^{*} \Phi=\beta^{2} R=\beta^{2} \mathcal{F} D \mathcal{F}^{*}
$$

where $\beta>0$.

The least-squares error $E$ of (66) and (67) may be expressed as

$$
\begin{aligned}
E & =\operatorname{Tr}\left((\Phi-F)^{*}(\Phi-F)\right) \\
& =\operatorname{Tr}\left(\Phi^{*} \Phi\right)+\operatorname{Tr}\left(F^{*} F\right)-2 \Re\left\{\operatorname{Tr}\left(F^{*} \Phi\right)\right\} \\
& =\beta^{2} \operatorname{Tr}(R)+\operatorname{Tr}\left(F^{*} F\right)-2 \Re\left\{\operatorname{Tr}\left(F^{*} \Phi\right)\right\} .
\end{aligned}
$$

Let $\tilde{\Phi}=(1 / \beta) \Phi$. Then minimizing $E$ is equivalent to minimizing

$$
E^{\prime}=\beta^{2} \operatorname{Tr}(R)-2 \beta \Re\left\{\operatorname{Tr}\left(F^{*} \tilde{\Phi}\right)\right\}
$$

subject to

$$
\tilde{\Phi}^{*} \tilde{\Phi}=R .
$$

To determine the optimal matrix $\Phi$ we have to minimize $E^{\prime}$ with respect to $\beta$ and $\tilde{\Phi}$. Fixing $\beta$ and minimizing with respect to $\tilde{\Phi}$, the optimal value of $\tilde{\Phi}$ is given by the SC-LSGUF of Section VIII-A with scaling $\beta_{0}=1$, so that

$$
\hat{\tilde{\Phi}}=U V^{*} \Sigma \mathcal{F}^{*} \text {. }
$$

If $F R F^{*}$ is invertible, then

$$
\hat{\tilde{\Phi}}=\left(F \mathcal{F} D \mathcal{F}^{*} F^{*}\right)^{-1 / 2} F \mathcal{F} D \mathcal{F}^{*}=\left(F R F^{*}\right)^{-1 / 2} F R \text {. }
$$

Substituting $\hat{\tilde{\Phi}}$ back into (74), and minimizing with respect to $\beta$, the optimal value of $\beta$ is

$$
\hat{\beta}=\frac{\Re\left\{\operatorname{Tr}\left(F^{*} \hat{\tilde{\Phi}}\right)\right\}}{\operatorname{Tr}(R)}=\frac{\operatorname{Tr}\left(U V^{*} \Sigma \mathcal{F}^{*}\right)}{\operatorname{Tr}(R)}
$$

which in the case that $F R F^{*}$ is invertible reduces to

$$
\hat{\beta}=\frac{\operatorname{Tr}\left(\left(F R F^{*}\right)^{1 / 2}\right)}{\operatorname{Tr}(R)} .
$$

The C-LSGUF vectors are then the columns of $\hat{\Phi}$ given by

$$
\hat{\Phi}=\hat{\beta} U V^{*} \Sigma \mathcal{F}^{*}
$$

where $\hat{\beta}$ is given by (78). If $F R F^{*}$ is invertible, then

$$
\hat{\Phi}=\hat{\beta}\left(F R F^{*}\right)^{-1 / 2} F R
$$

with $\hat{\beta}$ given by (79).

We summarize our results regarding constrained optimal GU frames in the following theorem.
Theorem $5(C-L S G U F)$ : Let $\left\{\varphi_{i}\right\}$ be a set of $n$ vectors in an $m$-dimensional Hilbert space $\mathcal{H}$ that span $\mathcal{H}$, and let $F$ be the matrix of columns $\varphi_{i}$. Let $\left\{\hat{\phi}_{i}\right\}$ denote the optimal $n \mathrm{GU}$ frame vectors that minimize the least-squares error defined by (66) and (67), subject to the constraint (68), and let $\hat{\Phi}$ be the matrix of columns $\phi_{i}$. Let $R$ be the matrix with $i$ th row equal to $P_{i}\left\{a_{j}, 1 \leq j \leq n\right\}$, so that $\beta^{2} R$ is the Gram matrix of inner products $\left\{\left\langle\phi_{i}, \phi_{j}\right\rangle\right\}$, and let $\mathcal{F}$ be the FT matrix that diagonalizes $R$. Let $D$ be the diagonal matrix with diagonal elements $\left\{\alpha_{j}=n^{1 / 2} \hat{a}_{j}, 1 \leq j \leq n\right\}$ where $\left\{\hat{a}_{j}, 1 \leq j \leq n\right\}$ is the FT of the sequence $\left\{a_{j}, 1 \leq j \leq n\right\}$, let $\Sigma$ be an $m \times n$ diagonal matrix with diagonal elements $\sqrt{\alpha_{i}}$ for values of $i$ for which $\alpha_{i} \neq 0$, and let $U$ and $V$ be the right-hand unitary matrix and left-hand unitary matrix, respectively, in the SVD of $F \mathcal{F} \Sigma^{*}$. Then

1) if $\beta=\beta_{0}$ is given, then $\hat{\Phi}=\beta_{0} U V^{*} \Sigma \mathcal{F}^{*}$. If in addition $F R F^{*}$ is invertible, then

$\hat{\Phi}=\beta_{0}\left(F \mathcal{F} D \mathcal{F}^{*} F^{*}\right)^{-1 / 2} F \mathcal{F} D \mathcal{F}^{*}=\beta_{0}\left(F R F^{*}\right)^{-1 / 2} F R$;

2) if $\beta>0$ is chosen to minimize $E$, then $\hat{\Phi}=\hat{\beta} U V^{*} \Sigma \mathcal{F}^{*}$, where $\hat{\beta}$ is given by (78). If, in addition, $F R F^{*}$ is invertible, then

$$
\hat{\Phi}=\hat{\beta}\left(F \mathcal{F} D \mathcal{F}^{*} F^{*}\right)^{-1 / 2} F \mathcal{F} D \mathcal{F}^{*}=\hat{\beta}\left(F R F^{*}\right)^{-1 / 2} F R
$$
where $\hat{\beta}$ is given by (79).

\section{Least-Squares GU Frame (LSGUF)}

We now consider the least-squares problem in which both the scaling factor $\beta$ and the numbers $\left\{a_{j}, 1 \leq j \leq n\right\}$ in (68) are chosen to minimize $E$. Thus, we seek a set of vectors $\left\{\phi_{i}\right\}$ that minimize the least-squares error $E$ of (66) and (67) subject to

$$
\left\{\left\langle\phi_{i}, \phi_{j}\right\rangle, 1 \leq j \leq n\right\}=P_{i}\left\{a_{j}, 1 \leq j \leq n\right\}
$$

where $P_{i}\left\{a_{j}, 1 \leq j \leq n\right\}$ is a known permutation of the unknown numbers $\left\{a_{j}, 1 \leq j \leq n\right\}$, chosen such that the matrix $R$ whose $i$ th row is equal to $P_{i}\left\{a_{j}, 1 \leq j \leq n\right\}$ is Hermitian, nonnegative definite, and diagonalized by an FT matrix $\mathcal{F}$.

This problem has been considered in the context of general least-squares inner product shaping [34], in which it was shown that the solution involves solving a problem of the form

$$
\max \sum_{i=1}^{m}\left|\left\langle x_{i}, y_{i}\right\rangle\right|^{2}
$$

subject to

$$
\left\langle y_{i}, y_{j}\right\rangle=\delta_{i j}
$$

where the vectors $\left\{x_{i}\right\}$ are known and are a function of the given vectors $\left\{\varphi_{i}\right\}$.

As we now show, this problem is equivalent to a detection problem in quantum mechanics, for which there is no known analytical solution in the general case. However, there exist very efficient computational methods for obtaining a numerical solution [39], [40].

1) Connection With Quantum Detection: In a quantum detection problem, a system is prepared in one of $m$ known (pure) states that are described by vectors $s_{i}$ in a Hilbert space $\mathcal{H}$, and the problem is to detect the state prepared by performing a measurement on the system. The measurement is described in terms of a set of orthogonal measurement vectors $q_{i}$. Given a set of 
measurement vectors $q_{i}$, and assuming equal prior probabilities on the different states, the probability of detection is given by [41]

$$
P_{q d}=\frac{1}{m} \sum_{i=1}^{m}\left|\left\langle q_{i}, s_{i}\right\rangle\right|^{2} .
$$

Comparing (85) with (83), we see that finding a set of orthonormal measurement vectors to maximize the probability of detection is equivalent to the maximization problem of (83) and (84).

Necessary and sufficient conditions for an optimum measurement maximizing (85) have been derived [42], [43], [41], [39]. However, except in some particular cases [41], [44], [45], [26], [46], obtaining a closed-form analytical expression for the optimal measurement directly from these conditions is a difficult and unsolved problem. Efficient iterative algorithms for maximizing (85) that are guaranteed to converge to the global optimum are given in [39], [40].

We conclude that in general there is no known analytical expression for the LSGUF. However, in practice the LSGUF can be approximated to any desired accuracy very efficiently using the iterative algorithms of [39], [40].

\section{Distance Properties of GU Frames}

So far, we have mainly been concerned with structural properties of GU frames. In this section, we study the Euclidean distance properties of these frames.

Suppose we are given a GU frame $\left\{\phi_{i}=U_{i} \phi, 1 \leq i \leq n\right\}$ generated by the group $\mathcal{Q}$ with $\|\phi\|^{2}=1$. We would like to characterize the distance profile $\alpha(i, j)=\left\|\phi_{i}-\phi_{j}\right\|^{2}$ for all $i, j$.

Since the vectors $\phi_{i}$ are $\mathrm{GU},\{\alpha(i, j), 1 \leq j \leq n\}$ is just a permutation of $\left\{d(i)=\left\|\phi-\phi_{i}\right\|^{2}, 1 \leq i \leq n\right\}$. Furthermore

$$
d(i)=\langle\phi, \phi\rangle+\left\langle U_{i} \phi, U_{i} \phi\right\rangle-2 \Re\left(\left\langle U_{i} \phi, \phi\right\rangle\right)=2\left(1-\Re\left(a_{i}\right)\right)
$$

where $\left\{a_{i}, 1 \leq i \leq n\right\}$ are the elements of the first row of the Gram matrix corresponding to the frame $\left\{\phi_{i}\right\}$.

In applications, it may be desirable to construct a $\mathrm{GU}$ frame such that $d(i)>0$ for $2 \leq i \leq n$. Since $d(i)=\left\|\left(I-U_{i}\right) \phi\right\|^{2}$, a sufficient condition is that

$$
\operatorname{det}\left(I-U_{i}\right) \neq 0, \quad 2 \leq i \leq n
$$

which is satisfied if and only if none of the matrices $U_{i}$ has an eigenvalue equal to 1 . Note that if (87) is satisfied, then $d(i)>0,2 \leq i \leq n$ regardless of the generating vector $\phi \neq 0$. Groups with unitary representations satisfying (87) are known as fixed-point free groups, and have been studied extensively in the literature (see, e.g., [47]). Thus, if $\mathcal{Q}$ is a representation of a fixed-point free group, then we have that $d(i)>0,2 \leq i \leq n$.

Fixed-point free groups have recently been studied in the context of unitary space-time codes [35]. In particular, it was shown in [35] that an Abelian group of matrices $\left\{U_{i}\right\}$ satisfies (87) if and only if it is cyclic, i.e., $U_{i}=U^{i}$ with $U^{n}=I$, and where $U$ can be parameterized as

$$
U=\operatorname{diag}\left(e^{j 2 \pi u_{1} / n}, \ldots, e^{j 2 \pi u_{n} / n}\right)
$$

where $u_{k}$ is relatively prime to $n$ for all $k$. An optimization over the $u_{k}$ can be performed to obtain distance profiles with certain prescribed properties (there are $\phi(n)$ positive integers less than $n$ that are relatively prime to $n$, where $\phi(n)$ denotes the Euler totient function of $n$ ).

\section{CONCLUSION}

In this paper, we introduced the concept of $\mathrm{GU}$ and $\mathrm{CGU}$ frames and discussed some of their key properties. A fundamental characteristic of these frames is that they possess strong symmetry properties that may be desirable in a variety of applications. In particular, similar to WH frames and wavelet frames, GU frames are generated by a single generating vector. Furthermore, the canonical and dual-frame vectors associated with a GU frame are themselves GU and are, therefore, also generated by a single generating vector which can be computed very efficiently using an FT matrix defined over an appropriate group.

We also showed that GU frame vectors exhibit interesting symmetry properties when one or more frame elements are removed. This property of GU frames may be of importance in multiple description source coding where it is often desirable that the quality of the reconstruction should not depend on the particular elements lost (removed).

Although in this paper we have focused on the case in which the underlying group is a finite Abelian group, many of the results can be extended to the more general case of infinite-dimensional and non-Abelian groups. An interesting direction for further research is to characterize these more general cases of GU frames using possibly continuous-time FTs defined over non-Abelian groups (see, e.g., [48]).

\section{ACKNOWLEDGMENT}

The authors wish to thank Prof. G. D. Forney for many fruitful discussions.

\section{REFERENCES}

[1] R. J. Duffin and A. C. Schaeffer, "A class of nonharmonic Fourier series," Trans. Amer. Math. Soc., vol. 72, pp. 314-366, 1952.

[2] R. M. Young, An Introduction to Nonharmonic Fourier Series. New York: Academic, 1980.

[3] C. E. Heil and D. F. Walnut, "Continuous and discrete wavelet transforms," SIAM Rev., vol. 31, no. 4, pp. 628-666, Dec. 1989.

[4] A. Aldroubi, "Portraits of frames," Proc. Amer. Math. Soc., vol. 123, pp. $1661-1668,1995$.

[5] J. J. Benedetto, "Irregular sampling and frames," in Wavelets-A Tutorial in Theory and Applications, C. K. Chui, Ed. Boca Raton, FL: CRC, 1992, pp. 445-507.

[6] I. Daubechies, "The wavelet transform, time-frequency localization and signal analysis," IEEE Trans. Inform. Theory, vol. 36, pp. 961-1005, Sept. 1990.

[7] H. Bölcskei, "Oversampled filter banks and predictive subband coders," Ph.D. dissertation, Vienna Univ. Technol., Vienna, Austria, Nov. 1997.

[8] H. Bölcskei, F. Hlawatsch, and H. G. Feichtinger, "Frame-theoretic analysis of oversampled filter banks," IEEE Trans. Signal Processing, vol. 46, pp. 3256-3268, Dec. 1998.

[9] Z. Cvetković and M. Vetterli, "Oversampled filter banks," IEEE Trans. Signal Processing, vol. 46, pp. 1245-1255, May 1998.

[10] P. J. S. G. Ferreira, "Mathematics for multimedia signal processing II-Discrete finite frames and signal reconstruction," in Signal Processing for Multimedia, J. S. Byrnes, Ed: IOC Press, 1999, pp. 35-54.

[11] A. Aldroubi and K. Gröchenig, "Non-uniform sampling and reconstruction in shift-invariant spaces," SIAM Rev., vol. 43, pp. 583-620, Dec. 2001. 
[12] Y. C. Eldar and A. V. Oppenheim, "Quantum signal processing," Signal Processing Mag., vol. 19, pp. 12-32, Nov. 2002.

[13] Y. C. Eldar and A. M. Chan, "An optimal whitening approach to linear multiuser detection," IEEE Trans. Inform. Theory, submitted for publication.

[14] V. K. Goyal, J. Kovačević, and J. A. Kelner, "Quantized frame expansions with erasures," Appl. Comp. Harmonic Analys., vol. 10, pp. 203-233, May 2001.

[15] D. Gabor, "Theory of communication," J. Inst. Elec. Eng., vol. 93, pp. 429-439, Nov. 1946.

[16] H. G. Feichtinger and T. Strohmer, Eds., Gabor Analysis: Theory, Algorithms, and Applications. Boston, MA: Birkhäuser, 1998.

[17] I. Daubechies, Ten Lectures on Wavelets. Philadelphia, PA: SIAM, 1992.

[18] D. Slepian, "Group codes for the Gaussian channel," Bell Syst. Tech. J., pp. 575-602, Apr. 1968.

[19] G. D. Forney, Jr., "Geometrically uniform codes," IEEE Trans. Inform. Theory, vol. 37, pp. 1241-1260, Sept. 1991.

[20] G. Ungerboeck, "Channel coding with multilevel/phase signals," IEEE Trans. Inform. Theory, vol. IT-28, pp. 55-67, Jan. 1982

[21] D. Raphaeli, "On multidimensional coded modulations having uniform error property for generalized decoding and flat-fading channels," IEEE Trans. Commun., vol. 46, pp. 34-40, Jan. 1998.

[22] G. Kaiser, A Friendly Guide to Wavelets. Boston, MA: Birkhauser, 1994.

[23] V. K. Goyal, J. Kovačević, and M. Vetterli, "Multiple description transform coding: Robustness to erasures using tight frame expansions," in Proc. IEEE Int. Symp. Information Theory, Cambridge, MA, Aug. 1998, p. 408.

[24] H. Bölcskei and A. J. E. M. Janssen, "Gabor frames, unimodularity, and window decay," J. Fourier Anal. Appl., vol. 6, no. 3, pp. 255-276, 2000.

[25] A. J. E. M. Janssen and H. Bölcskei, "Equivalence of two methods for constructing tight Gabor frames," IEEE Signal Processing Lett., vol. 7, pp. 79-82, Apr. 2000.

[26] Y. C. Eldar and G. D. Forney, Jr., "On quantum detection and the square-root measurement," IEEE Trans. Inform. Theory, vol. 47, pp. 858-872, Mar. 2001.

[27] A. J. E. M. Janssen and T. Strohmer, "Characterization and computation of canonical tight windows for Gabor frames," J. Fourier Anal. Applic., vol. 8, no. 1, pp. 1-28, 2002.

[28] Y. Meyer, "Ondelettes et functions splines," in Seminaire EDP. Paris, France, Dec. 1986.

[29] Y. C. Eldar and G. D. Forney, Jr., "Optimal tight frames and quantum measurement," IEEE Trans. Inform. Theory, vol. 48, pp. 599-610, Mar. 2002.

[30] T. Strohmer, "Finite and infinite-dimensional models for oversampled filter banks," in Modern Sampling Theory: Mathematics and Applications, J. J. Benedetto and P. J. S. G. Ferreira, Eds. Boston, MA: Birkhäuser, 2000, pp. 297-320.
[31] I. Daubechies, "Orthonormal bases of compactly supported wavelets," Comm. Pure Appl. Math., vol. 41, pp. 909-996, 1988.

[32] S. G. Mallat, "A theory of multiresolution signal decomposition: The wavelet representation," IEEE Trans. Pattern Anal. Machine Intell., vol. 11, pp. 674-693, July 1989.

[33] M. Unser and A. Aldroubi, "Families of multiresolution and wavelet spaces with optimal properties," Numer. Funct. Anal. Optimiz., vol. 14, pp. 417-446, 1993.

[34] Y. C. Eldar, "Least-squares inner product shaping," Linear Algebra Appl., vol. 348, pp. 153-174, May 2002

[35] A. Shokrollahi, B. Hassibi, B. M. Hochwald, and W. Sweldens, "Representation theory for high-rate multiple-antenna code design," IEEE Trans. Inform. Theory, vol. 47, pp. 2335-2367, Sept. 2001.

[36] A. Ben-Israel and T. N. E. Greville, Generalized Inverses: Theory And Applications. Huntington, NY: Krieger, 1980.

[37] M. A. Armstrong, Groups and Symmetry. New York: Springer-Verlag, 1988.

[38] G. H. Golub and C. F. Van Loan, Matrix Computations, 3rd ed. Baltimore, MD: Johns Hopkins Univ. Press, 1996.

[39] Y. C. Eldar, A. Megretski, and G. C. Verghese, "Designing optimal quantum detectors via semidefinite programming," IEEE Trans. Inform. Theory, vol. 49, pp. 1007-1012, Apr. 2003.

[40] Y. C. Eldar, "Least-squares orthogonalization using semidefinite programming," Linear Algebra Appl., submitted for publication.

[41] C. W. Helstrom, Quantum Detection and Estimation Theory. New York: Academic, 1976.

[42] A. S. Holevo, "Statistical decisions in quantum theory," J. Multivar. Anal., vol. 3, pp. 337-394, Dec. 1973.

[43] H. P. Yuen, R. S. Kennedy, and M. Lax, "Optimum testing of multiple hypotheses in quantum detection theory," IEEE Trans. Inform. Theory, vol. IT-21, pp. 125-134, Mar. 1975.

[44] M. Charbit, C. Bendjaballah, and C. W. Helstrom, "Cutoff rate for the $m$-ary PSK modulation channel with optimal quantum detection," IEEE Trans. Inform. Theory, vol. 35, pp. 1131-1133, Sept. 1989.

[45] M. Ban, K. Kurokawa, R. Momose, and O. Hirota, "Optimum measurements for discrimination among symmetric quantum states and parameter estimation," Int. J. Theor. Phys., vol. 36, pp. 1269-1288, 1997.

[46] Y. C. Eldar, A. Megretski, and G. C. Verghese. Optimal detection of symmetric mixed quantum states. [Online]. Available: http://www.arXiv. org/abs/quant-ph/0 211111.

[47] G. James and M. Liebeck, Representations and Characters of Groups. Cambridge, U.K.: Cambridge Univ. Press, 1993.

[48] A. Terras, Fourier Analysis on Finite Groups and Applications. Cambridge, U.K.: Cambridge Univ. Press, 1999. 